\title{
Detection of coherent low-frequency radio bursts from weak-line T Tauri stars
}

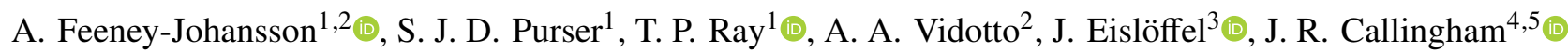 \\ T. W. Shimwell ${ }^{5,4}$, H. K. Vedantham ${ }^{6}$, G. Hallinan ${ }^{7}$, and C. Tasse ${ }^{8,9}$ \\ ${ }^{1}$ Dublin Institute for Advanced Studies, Astronomy \& Astrophysics Section, 31 Fitzwilliam Place, Dublin D02 XF86, Ireland \\ e-mail: antonfj@cp.dias.ie \\ 2 School of Physics, Trinity College Dublin, College Green, Dublin 2, Ireland \\ 3 Thüringer Landessternwarte Tautenburg, Sternwarte 5, 07778 Tautenburg, Germany \\ ${ }^{4}$ Leiden Observatory, Leiden University, PO Box 9513, 2300 RA Leiden, The Netherlands \\ 5 ASTRON, Netherlands Institute for Radio Astronomy, Oude Hoogeveensedijk 4, Dwingeloo 7991 PD, The Netherlands \\ ${ }^{6}$ Kapteyn Astronomical Institute, University of Groningen, PO Box 72, 97200 AB Groningen, The Netherlands \\ 7 Cahill Center for Astronomy and Astrophysics, California Institute of Technology, Pasadena, CA 91125, USA \\ ${ }^{8}$ GEPI \& USN, Observatoire de Paris, CNRS Université Paris Diderot, 5 place Jules Janssen, 92190 Meudon, France \\ 9 Centre for Radio Astronomy Techniques and Technologies, Department of Physics and Electronics, Rhodes University, \\ Grahamstown 6140, South Africa
}

Received 22 March 2021 / Accepted 27 June 2021

\section{ABSTRACT}

\begin{abstract}
In recent years, thanks to new facilities such as LOFAR that are capable of sensitive observations, much work has been done on the detection of stellar radio emission at low frequencies. Such emission has commonly been shown to be coherent emission, generally attributed to electron-cyclotron maser (ECM) emission, and has usually been detected from main-sequence M dwarfs. Here we report the first detection of coherent emission at low frequencies from $\mathrm{T}$ Tauri stars, which are known to be associated with high levels of stellar activity. Using LOFAR, we detect several bright radio bursts at $150 \mathrm{MHz}$ from two weak-line T Tauri stars: KPNO-Tau 14 and $\mathrm{LkCa} 4$. All of the bursts have high brightness temperatures $\left(10^{13}-10^{14} \mathrm{~K}\right)$ and high circular polarisation fractions $(60-90 \%)$, indicating that they must be due to a coherent emission mechanism. This could be either plasma emission or ECM emission. Due to the exceptionally high brightness temperatures seen in at least one of the bursts $\left(\geq 10^{14} \mathrm{~K}\right)$, as well as the high circular polarisation levels, it seems unlikely that plasma emission could be the source; as such, ECM is favoured as the most likely emission mechanism. Assuming this is the case, the required magnetic field in the emission regions would be $40-70 \mathrm{G}$. We determine that the most likely method of generating ECM emission is plasma co-rotation breakdown in the stellar magnetosphere. There remains the possibility, however, that it could be due to an interaction with an orbiting exoplanet.
\end{abstract}

Key words. stars: pre-main sequence - stars: low-mass - stars: individual: KPNO-Tau 14 - stars: individual: LkCa 4 radio continuum: stars

\section{Introduction}

T Tauri stars (TTSs) are low-mass $\left(<2 M_{\odot}\right)$ young stellar objects (YSOs) that have evolved to the state where they are optically visible. They are frequently associated with magnetic activity seen in the form of flaring and magnetospheric accretion bursts (Feigelson \& Montmerle 1999). T Tauri stars can be divided into two categories: classical T Tauri stars (CTTSs), which are surrounded by a circumstellar accretion disk, and weak-line T Tauri stars (WTTSs), where most, if not all, of the disk has dissipated. Flares from these objects are thought to be caused by magnetic reconnection events, similar to the ones observed on the Sun but with energies up to $10^{6}$ times larger (e.g., Fernández et al. 2004). In addition, while reconnection in the Sun and other main sequence stars occurs in magnetic fields anchored in the star, reconnection in YSOs can occur in magnetic fields connecting the star and the disk (Waterfall et al. 2019).

Flaring of WTTSs has been observed at a variety of wavelengths, from the X-ray (e.g., Uzawa et al. 2011) to radio regimes (e.g., Forbrich et al. 2017). In the radio band, observations have normally been made at centimetre wavelengths $(\geq 1 \mathrm{GHz})$, where any detected emission is generally attributed to incoherent gyro- synchrotron emission from energetic electrons excited in magnetic reconnection events (Waterfall et al. 2019).

Among main sequence stars, radio bursts have primarily been observed from $\mathrm{M}$ dwarfs, which are known to be magnetically active (Villadsen \& Hallinan 2019). At frequencies below or around $1 \mathrm{GHz}$, such bursts have been found to be coherent emission, generated by either plasma emission or electroncyclotron maser (ECM) emission. In both cases, coherent radio emission is generally characterised by very high brightness temperatures (Dulk 1985).

For example, one well-known flare star, UV Ceti, was detected at $154 \mathrm{MHz}$ using the Murchison Widefield Array (Lynch et al. 2017). This emission was determined to be coherent based on its high brightness temperature $\left(10^{13}-10^{14} \mathrm{~K}\right)$ and, most likely, ECM emission due to the presence of elliptical polarisation, which is thought to only be generated in the $\mathrm{x}$ mode (Dulk et al. 1994). From theory, it is predicted that ECM emission is entirely polarised in the $\mathrm{x}$ mode, while plasma emission is polarised in the o mode (Dulk 1985).

In addition, Zic et al. (2019) detected several periodic, coherent bursts from UV Ceti. The period of the bursts is consistent with the rotation period of the star, and, based on the presence 
Table 1. Primary characteristics of KPNO-Tau 14 and LkCa 4.

\begin{tabular}{lcc}
\hline \hline & KPNO-Tau 14 & LkCa 4 \\
\hline Spectral type & M6 & K7 \\
Age (Myr) & $1^{1}$ & $2^{6} / 0.5^{7}$ \\
Distance $(\mathrm{pc})$ & $151.9_{-4.12}^{+5.8}$ & $129.4 \pm 0.6^{2}$ \\
Mass $\left(M_{\odot}\right)$ & $0.1^{1}$ & $0.79 \pm 0.05^{6} / 0.15-0.30^{7}$ \\
Radius $\left(R_{\odot}\right)$ & $\sim 1^{3}$ & $2.3^{7}$ \\
Rotation period & $1.86 \pm 0.01^{4}$ & $3.37 \pm 0.01^{8}$ \\
Surface magnetic field strength $(\mathrm{kG})$ & - & $1.6^{6}$ \\
Coronal temperature $(\mathrm{MK})$ & $12.6^{5}$ & - \\
\hline
\end{tabular}

Notes. Due to the uncertainty in the age and mass of $\mathrm{LkCa} 4$, two values are given for these parameters.

References. (1) Luhman et al. (2003); (2) Bailer-Jones et al. (2018); (3) Derived from Baraffe et al. (2015) models; (4) Scholz et al. (2018); (5) Güdel et al. (2007); (6) Donati et al. (2014); (7) Gully-Santiago et al. (2017); (8) Grankin et al. (2008).

of high brightness temperatures and elliptical polarisation, the bursts were determined to be strongly beamed ECM emission originating from a low-density cavity within the magnetosphere of UV Ceti. This illustrates that auroral emission is possible in main sequence $M$ dwarfs, similar to that seen in brown dwarfs and Solar System planets (Pineda et al. 2017; Zarka 1998).

Recently, coherent radio emission was detected from the flare star CR Draconis by Callingham et al. (2021) using the Low-Frequency Array (LOFAR; Van Haarlem et al. 2013). When observed for a total of 6.5 days over one year, three bright radio bursts were detected in addition to an almost steady radio flux. Due to the high brightness temperatures, it was determined that the emission must be coherent. Moreover, it shows high levels of left-handed circular polarisation at all epochs. The consistent handedness of the circular polarisation indicates that the emission originates from a region with the same magnetic polarity. Thus, it is unlikely to be due to plasma emission generated by flares since the polarity would be expected to flip due to flares occurring across the stellar disk. In addition, elliptical polarisation was also seen in one of the detected bursts. Based on these factors, it was determined that the emission must be ECM emission.

However, very little work has been done on searching for radio emission from YSOs at low $(<1 \mathrm{GHz})$ radio frequencies. The detection of such emission can give us important information on the presence of any high energy particles and magnetic fields in YSO outflows (Ainsworth et al. 2014; FeeneyJohansson et al. 2019), provide evidence for possible star-planet interactions (Vedantham et al. 2020; Kavanagh et al. 2021), and help evaluate the importance of coronal mass ejection (CME) events in dissipating proto-planetary disks (Schnepf et al. 2015).

While in its infancy, the study of YSOs at low radio frequencies is nevertheless a growing field thanks to new facilities such as LOFAR. Now one can utilise not only single target observations but also surveys such as the LOFAR Two-metre Sky Survey (LoTSS), which allow steady and transient sources to be found (Shimwell et al. 2017).

With these ideas in mind, we have started an initial study of the Taurus Molecular Cloud (TMC) using the High-Band Array (HBA) of LOFAR centred on approximately $150 \mathrm{MHz}$. It should be noted that the HBA was used for this study to take advantage of its much higher sensitivity compared to the LOFAR LowBand Array (LBA). Here we discuss two WTTSs in the TMC, KPNO-Tau 14 and $\mathrm{LkCa} 4$, the primary characteristics of which are listed in Table 1.
KPNO-Tau 14 is a very low-mass $\left(0.1 M_{\odot}\right)$ WTTS of spectral type M6 (Luhman et al. 2003). This puts the star very close to the brown dwarf upper limit $\left(<0.08 M_{\odot}\right)$. Based on Gaia measurements (Gaia Collaboration 2018), its distance is $151.9_{-4.8}^{+5.1} \mathrm{pc}$ (Bailer-Jones et al. 2018). No significant level of accretion is detected in this YSO and there is no infrared excess in its spectrum (Muzerolle et al. 2005; Mohanty et al. 2005), suggesting that there is no dusty disk present. Mohanty et al. (2005) found evidence that KPNO-Tau 14 may be a spectroscopic binary, based on the measurement of a double peak in the cross-correlation profile of its $\mathrm{H} \alpha$ line. However, a highresolution imaging survey by Kraus et al. (2006) using the Hubble Space Telescope (HST) was unable to resolve the system, giving an upper limit for the projected binary separation of $<4$ au. Magnetic activity has been inferred from KPNO-Tau 14. More precisely, X-ray emission was detected from this star in the XMM-Newton extended survey of the Taurus Molecular Cloud (XEST) by Güdel et al. (2007). During these observations, an $\mathrm{X}$-ray flare was found with a luminosity of $1.58 \times 10^{30} \mathrm{erg} \mathrm{s}^{-1}$ (corrected for extinction) and a duration of $>6000$ s (Stelzer et al. 2007). For comparison, the median X-ray luminosity for TTSs detected by Güdel et al. (2007) was $0.7 \times 10^{30} \mathrm{erg} \mathrm{s}^{-1}$.

LkCa 4 is another WTTS of spectral type K7. Based on Gaia, the distance to this star is $129.4 \pm 0.6 \mathrm{pc}$ (Bailer-Jones et al. 2018). Like KPNO-Tau 14, it has no infrared excess, indicating that its dusty disk has dissipated or at least that significant grain growth has occurred, thus reducing its infrared emission (Esplin et al. 2014). LkCa 4 is known to be very magnetically active. This is deduced from its high level of starspot coverage and intense flaring. In fact, Gully-Santiago et al. (2017) discovered, using model atmospheres, that its spectrum is best fitted with two temperature components: a hot photosphere of $T_{\text {hot }} \sim 4100 \mathrm{~K}$ covering $20 \%$ of the surface and a cool component with $T_{\text {cool }} \sim 2700-3000 \mathrm{~K}$ accounting for the remainder. This suggests a remarkable starspot coverage of $80 \%$. Flaring activity was previously seen at radio frequencies by Dzib et al. (2015), who observed mildly circularly polarised emission at 4.5 GHz and 7.5 GHz. The emission is highly variable in Stokes $I$ over three epochs, with changes of $92.7 \pm 22.7 \%$ at $4.5 \mathrm{GHz}$ and $90.8 \pm 20.1 \%$ at $7.5 \mathrm{GHz}$. The circular polarisation fractions measured are $9.6 \%$ at $4.5 \mathrm{GHz}$ and $18.8 \%$ at $7.5 \mathrm{GHz}$. LkCa 4 was also detected in X-rays by Chandra with a measured luminosity corrected for extinction of $2.0 \times 10^{30} \mathrm{erg} \mathrm{s}^{-1}$ (Yang et al. 2012).

The magnetic field of $\mathrm{LkCa} 4$ was modelled by Donati et al. (2014), who found that it possesses a strong and mainly 
Table 2. Details of the LOFAR observations of KPNO-Tau 14.

\begin{tabular}{lccccccc}
\hline \hline Project code & LoTSS pointing & Date & Time & $\begin{array}{c}\text { Duration } \\
(\mathrm{h})\end{array}$ & $\begin{array}{c}v \\
(\mathrm{MHz})\end{array}$ & $\begin{array}{c}\Delta v \\
(\mathrm{MHz})\end{array}$ & $\begin{array}{c}\sigma_{\mathrm{rms}} \\
(\mu \mathrm{Jy})\end{array}$ \\
\hline LC1_001 & - & $2013-11-30$ & $19: 15: 00$ & 8.0 & 152 & 74 & 210 \\
L12_015 & P068+26 & $2019-06-28$ & $05: 11: 00$ & 8.0 & 144 & 48 & 130 \\
\hline
\end{tabular}

Notes. The Date and Time columns refer to the time in UTC at the start of each observation. $v$ is the central frequency of the observation, and $\Delta v$ is the bandwidth. $\sigma_{\text {rms }}$ is the root-mean-square noise in Stokes $I$ at the position of KPNO-Tau 14.

axisymmetric magnetic field with a poloidal component of $\approx 2 \mathrm{kG}$ and a toroidal component of $\approx 1 \mathrm{kG}$. There is a large degree of uncertainty regarding the mass and age of $\mathrm{LkCa} 4$ due to its high level of starspot coverage. While previous estimates, using an effective temperature of $4000 \mathrm{~K}$, had found mass and age estimates of $0.79 \pm 0.05 M_{\odot}$ and $2 \mathrm{Myr}$, Gully-Santiago et al. (2017) find an effective temperature of $3180 \mathrm{~K}$ based on their two-temperature component model. Comparing this to stellar evolutionary models (Baraffe et al. 2015) gives mass and age estimates of $0.15-0.30 M_{\odot}$ and $\sim 0.5 \mathrm{Myr}$, respectively. These evolutionary models assume the star to be unspotted, however, and thus there remain major uncertainties.

In this paper we report the serendipitous detection of bright radio bursts at low frequencies from these two sources using LOFAR. In Sect. 2 we describe how the observations were carried out and the data reduction process. In Sect. 3 we show the resulting images and light curves of the observations that were obtained and describe the characteristics of the bursts that were detected. In Sect. 4 we then discuss which of the possible emission mechanisms can explain the observations. In Sect. 5 we discuss whether the ECM mechanism could also be observed from YSOs at higher radio frequencies. Finally, in Sect. 6 we present our concluding remarks.

\section{Observations and data reduction}

\subsection{LOFAR observations}

As part of a project to search for emission from the jets of low-mass YSOs (LOFAR project code: LC1_001), the CTTS DG Tau A, located in the TMC, was observed using the HBA on 2013 November 30-December 1 (epoch 2013.91). The total observing time was $8 \mathrm{~h}$ with a central frequency of $152 \mathrm{MHz}$ and a bandwidth of $74 \mathrm{MHz}$ (Feeney-Johansson et al. 2019). Details of this observation are given in Table 2.

Due to the ionosphere and imperfect knowledge of beam shapes, there are important direction-dependent effects (DDEs) present in LOFAR data that must be removed. Directionindependent calibration was first performed using the LOFAR calibration pipeline PREFACTOR ${ }^{1}$ described by de Gasperin et al. (2019). Subsequent direction-dependent calibration was carried out using the facet calibration pipeline FACTOR ${ }^{2}$ described by Van Weeren et al. (2016), followed by imaging using WSClean (Offringa et al. 2014). The resolution achieved in the final image is $5.88^{\prime \prime} \times 5.15^{\prime \prime}$. Full details of the process are given in FeeneyJohansson et al. (2019).

Due to inaccuracies in LOFAR beam models (Van Weeren et al. 2016; Shimwell et al. 2019), the derived flux density scale is often not accurate. To account for this, in FeeneyJohansson et al. (2019) the integrated flux densities of several compact bright sources in the field were compared with their

\footnotetext{
1 https://github.com/lofar-astron/prefactor

2 https://github.com/lofar-astron/factor
}

values in the TIFR GMRT Sky Survey (TGSS; Intema et al. 2017). It was found that the average ratio of TGSS flux densities to LOFAR flux densities was $0.83 \pm 0.17$. However, there are known to be significant uncertainties with the flux density scale of TGSS. For this reason, and also to be consistent with the 2019 observations in the next section, the correction factor for the flux density scale was re-derived using the NRAO VLA Sky Survey (NVSS; Condon et al. 1998) and the 6C catalogue (Hales et al. 1988). This technique is described in more detail in Hardcastle et al. (2021). The flux-scale correction derived is $1.04 \pm 0.21$ and so the flux densities measured in the LOFAR image were corrected by this factor. Shimwell et al. (2019) found that there is still, conservatively, a $20 \%$ error in the flux density scale of LOFAR images after correction. Therefore, there is estimated to be an error of \pm 0.21 in the correction factor.

Although the main objective was to detect emission from the vicinity of DG Tau A, the full field was examined for other YSOs. A catalogue of all radio sources down to a $5 \sigma$ signal-tonoise threshold was extracted using PyBDSF (Mohan \& Rafferty 2015), which was then compared with those of known YSOs in XEST (Güdel et al. 2007), the Gould Belt GHz Very Large Array (VLA) Survey (Dzib et al. 2015), the Taurus Spitzer Survey (Rebull et al. 2010), and the Taurus WISE Survey (Esplin et al. 2014).

Out of a total of 115 YSOs within the field, radio emission was detected at the position of 2 other YSOs in addition to DG Tau A: KPNO-Tau 14 and V1320 Tau, with the noise level ranging from $100 \mu \mathrm{Jy}_{\text {beam }}{ }^{-1}$ at the centre of the field to $400 \mu \mathrm{Jy}_{\text {beam }^{-1}}$ near the edge of the field. While the flux of V1320 Tau is steady, KPNO-Tau 14 shows strong flaring during our observing run. As this paper focuses on radio bursts, V1320 Tau will be discussed later in a separate paper on lowfrequency emission from YSOs in the TMC (Feeney-Johansson et al., in prep.).

\subsection{LOFAR observations}

The LOFAR Two-Metre Sky Survey (LoTSS) is mapping the entire northern sky at $120-168 \mathrm{MHz}$ with a target sensitivity of $\sim 100 \mu_{\mathrm{Jy}}$ beam $^{-1}$ (Shimwell et al. 2017). As part of this survey, the Taurus and Perseus Molecular Clouds were imaged in a co-observing proposal (LOFAR Project code: LC12_015) in a series of observations during LOFAR Cycle 12, from JuneNovember 2019. These regions each contain at least 414 and 369 YSOs, respectively (Esplin et al. 2014; Young et al. 2015). Details of these observations are given in Tables 2 and 3. Work on this survey is currently ongoing and the full results will be published in a future paper.

Direction-independent calibration was carried out on each of the observations using the PREFACTOR pipeline. To correct for DDEs, the data were processed using the DDF Pipeline (Shimwell et al. 2019; Tasse et al. 2021), which uses the KillMS 
Table 3. Details of the LOFAR observations of $\mathrm{LkCa} 4$.

\begin{tabular}{lcccccccc}
\hline \hline Project code & LoTSS pointing & Date & Time & $\begin{array}{c}\text { Duration } \\
(\mathrm{h})\end{array}$ & $\begin{array}{c}v \\
(\mathrm{MHz})\end{array}$ & $\begin{array}{c}\Delta v \\
(\mathrm{MHz})\end{array}$ & $\begin{array}{c}\sigma_{\text {rms }} \\
(\mu \mathrm{Jy})\end{array}$ & Phase \\
\hline L12_015 & P061+29 & $2019-06-28$ & $05: 11: 00$ & 8.0 & 144 & 48 & 230 & $0.00-0.10$ \\
L12_015 & P062+26 & $2019-07-06$ & $04: 52: 15$ & 8.0 & 144 & 48 & 240 & $0.37-0.47$ \\
L12_015 & P065+26 & $2019-07-07$ & $04: 59: 59$ & 8.0 & 144 & 48 & 280 & $0.67-0.77$ \\
L12_015 & P064+29 & $2019-08-10$ & $02: 11: 00$ & 8.0 & 144 & 48 & 150 & $0.71-0.81$ \\
\hline
\end{tabular}

Notes. The Date and Time columns refer to the time in UTC at the start of each observation. $v$ is the central frequency of the observation, and $\Delta v$ is the bandwidth. $\sigma_{\text {rms }}$ is the root-mean-square noise in Stokes $I$ at the position of LkCa 4 . The rotation phase is calculated using the known rotation period of LkCa 4 of $3.37 \mathrm{~d}$ (Grankin et al. 2008) such that phase 0.0 corresponds to the beginning of the observation of LoTSS pointing P061+29 (2019-06-28 05:11:00).

package (Smirnov \& Tasse 2015) to solve for the DDEs and then uses DDFacet (Tasse et al. 2018), which applies the DDEs while imaging $^{3}$. The final output was a full-bandwidth Stokes $I$ image of the field with a resolution of $6^{\prime \prime}$. In addition, a low-resolution $20^{\prime \prime}$ Stokes $V$ image was also obtained.

As with the 2013 observations, and for each pointing, we extracted a list of sources for comparison with the previously cited YSO catalogues. In pointing P064+29, carried out on August 10, 2019, emission was detected towards LkCa 4. Similar to KPNO-Tau 14, this source also showed very strong flaring over the observing period. The position of $\mathrm{LkCa} 4$ was also included in three other pointings: P061+29, P062+26, and P065+26, dated June 28, July 6, and July 7, 2019, respectively. However, no emission was detected towards $\mathrm{LkCa} 4$ in any of these earlier epochs. Likewise, the position of KPNO-Tau 14 was included in the pointing P068+26, dated June 28, 2019, but no emission was detected.

Similar to the 2013 LOFAR observations, the flux density scales for each of the LoTSS pointings were corrected due to errors in the beam models by deriving correction factors using NVSS and the 6C catalogue. For P064+29, the flux-scale correction derived was $1.24 \pm 0.25$ and so the flux density measurements in this pointing were multiplied by this factor. Again, based on Shimwell et al. (2019) there was assumed to be an error of $20 \%$ in this correction factor.

\section{Results}

\subsection{KPNO-Tau 14}

In the 2013 observations, emission is detected from the position of KPNO-Tau 14 with a peak flux density (Stokes I) of $3.52 \pm$ $0.73 \mathrm{mJy}$ beam $^{-1}$, as seen in Fig. 1 . In addition, emission is also detected with a peak flux density of $3.28 \pm 0.67 \mathrm{mJy} \mathrm{beam}^{-1}$ in Stokes $V$, showing that the emission is highly circularly polarised with a polarisation fraction of $80_{-17}^{+13} \%$. Given that the source is unresolved, we take the peak flux density to be the total flux density.

The error in the flux density measurement, $\sigma_{S_{v}}$, and subsequent flux density measurements in this paper, was taken to be a combination of the root-mean-square noise $\sigma_{\text {rms }}$ of the image and the absolute flux calibration error, found by Shimwell et al. (2019) to be $20 \%$ for LOFAR after applying a correction factor based on other surveys: $\sigma_{S_{v}}=\sqrt{\sigma_{\text {rms }}^{2}+\left(0.20 \times S_{v}\right)^{2}}$. The polarisation fraction values and errors for this and all subsequent flux

\footnotetext{
3 See https://github.com/saopicc for the KillMS and DDFacet packages and https://github.com/mhardcastle/ddf-pipeline for the DDF-Pipeline.
}

density measurements were found as follows. Gaussian distributions were generated for the Stokes $I$ and Stokes $V$ values, with a mean of the measured flux value $S_{v}$ and a standard deviation of $\sigma_{S_{v}}$. The values in the Stokes $V$ distribution were divided by the values in the Stokes $I$ distribution to generate a distribution of polarisation fraction values. This polarisation fraction distribution was then truncated above $100 \%$ to remove any values higher than this. The value of the polarisation fraction was then taken to be the median of this truncated distribution and the errors give the $68 \%$ confidence interval.

To investigate time variability, the direction-dependent calibrated data were divided into time bins of $30 \mathrm{~min}$. Each period was then imaged using WSClean in Stokes $I$ and $V$. To check that the flux density scale calibration was accurate for all the images produced, the flux densities of several nearby compact bright sources in the field were measured in each image using PyBDSF and their light curves were plotted to check for any variation in the measured flux densities (see Appendix A for the light curves). Over the $8 \mathrm{~h}$, the flux densities of these sources vary with an average standard deviation of $12 \%$ relative to the mean flux density of each source. Given that this is within the typical absolute flux calibration error for LOFAR, we conclude that the flux density scale calibration is accurate for all of the images.

It was found that the flux density from KPNO-Tau 14 varied significantly over the $8 \mathrm{~h}$ of observation. This can be seen in the light curve in Fig. 2 where 2 bursts can be clearly seen. In particular, the first burst, $1.5-2 \mathrm{~h}$ after the start of our observation, is very strong with a flux density of $11.25 \pm 1.15 \mathrm{mJy}$. We note that the errors for this light curve and the subsequent light curves in this paper do not take into account the absolute flux calibration error as any absolute flux calibration offset should be the same for the whole observation.

In order to study the first burst in more detail, the data were further sub-divided into 3-min time bins and plotted in the light curve seen in Fig. 3, showing that the duration of the first burst is approximately $15 \mathrm{~min}$ and the flux density peaks at $28.15 \pm 3.02 \mathrm{mJy}$. It should be noted that the true maximum flux density may be higher but due to our time binning, this may have been averaged out. Unfortunately, we cannot obtain a higher time resolution as the signal-to-noise would be too poor.

While the second burst seen in Fig. 2 is not as strong as the first, it has a longer duration, lasting from $2.5 \mathrm{~h}$ to $4.5 \mathrm{~h}$ after the start of the observation, and peaking at $8.70 \pm 0.87 \mathrm{mJy}$. Some varying low-level emission is then detected during the rest of the observing window. The emission is highly circularly polarised throughout, with the exception of that detected at $5.0-5.5 \mathrm{~h}$, and is consistently left-hand circularly polarised (see Appendix B). Both of the major bursts detected are strongly 

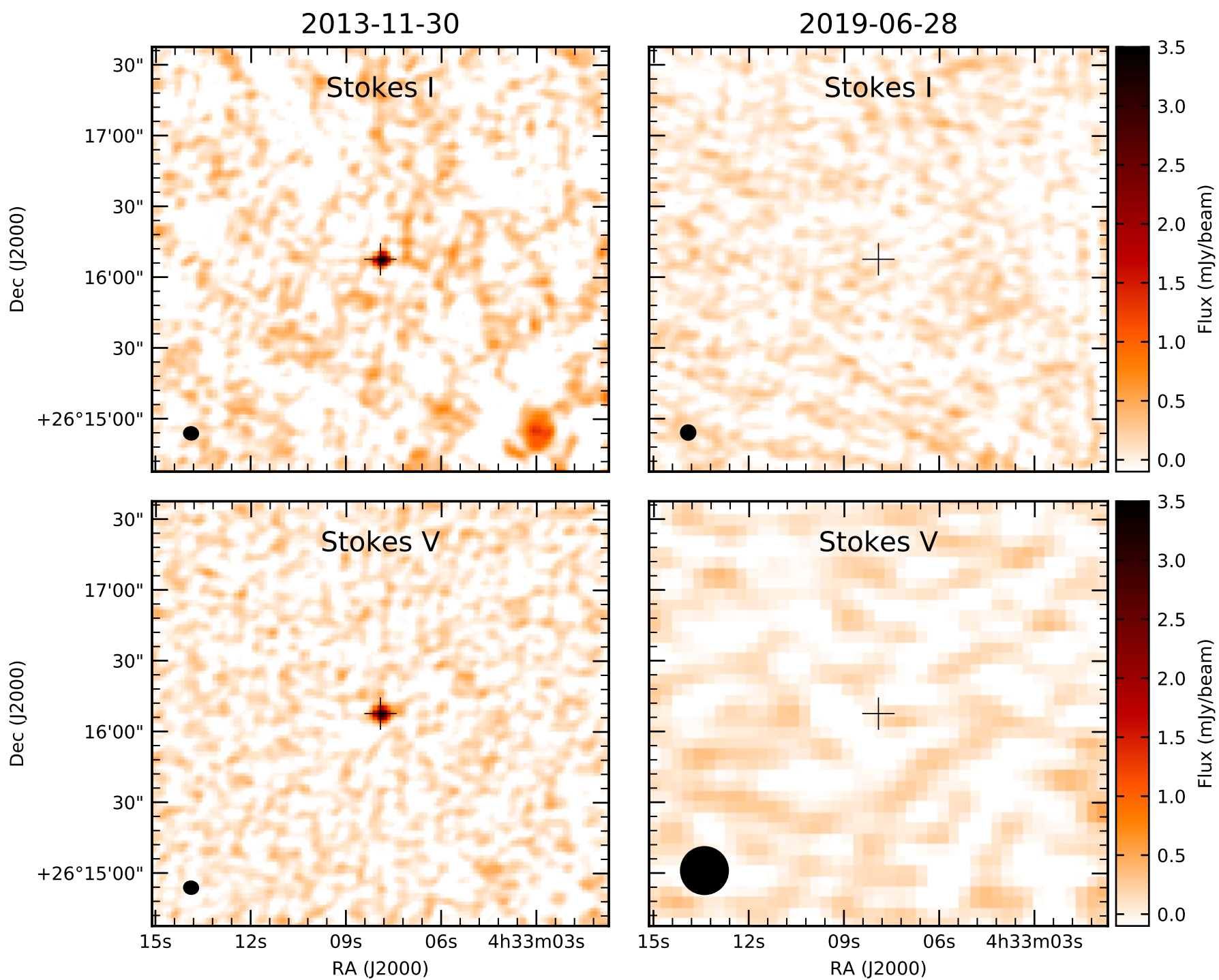

Fig. 1. Stokes $I$ (top) and Stokes $V$ (bottom) images of KPNO-Tau 14 obtained from LOFAR at at two epochs: November 30, 2013 (left) and June 28, 2019 (right). The restoring beam used for the 2013 images is $5.88^{\prime \prime} \times 5.15^{\prime \prime}$. The restoring beams used for the 2019 LoTSS images are $6^{\prime \prime} \times 6^{\prime \prime}$ and $20^{\prime \prime} \times 20^{\prime \prime}$ for the Stokes $I$ and Stokes $V$ images, respectively. The optical position of KPNO-Tau 14 obtained from Gaia DR2 (Gaia Collaboration 2018) is indicated by the cross.

circularly polarised, with polarisation fractions of $88_{-9}^{+8} \%$ and $91_{-8}^{+6} \%$, respectively.

Based on the distance to the source, and assuming the emission is isotropic (i.e., not beamed), the first and largest burst has a peak luminosity of at least $(7.8 \pm 1.0) \times 10^{17} \mathrm{erg} \mathrm{s}^{-1} \mathrm{~Hz}^{-1}$, while the second smaller burst has a peak luminosity of at least $(2.4 \pm 0.3) \times 10^{17} \mathrm{erg} \mathrm{s}^{-1} \mathrm{~Hz}^{-1}$. To calculate the corresponding brightness temperatures, we assume, as indicative, the size of the emission region to be a circular disk with a radius equal to the radius of the star. From the Baraffe et al. (2015) evolutionary models for TTSs, a $0.1 M_{\odot}$ star with an age of $1 \mathrm{Myr}$ should have a radius of $\sim 1 R_{\odot}$. This would mean that the brightness temperatures of the first and second bursts are at least $(5.8 \pm 0.7) \times 10^{14} \mathrm{~K}$ and $(1.8 \pm 0.2) \times 10^{14} \mathrm{~K}$, respectively. However, given that the emission region is likely much smaller than the entire stellar surface, we are almost certainly underestimating the actual brightness temperatures.

Finally, we remark that the presence of strong circular polarisation and the transient nature of the emission implies that the emission is associated with KPNO-Tau 14 and not a background extragalactic source for example. We also note that no emission is detected in either Stokes $I$ or $V$ from KPNO-Tau 14 in the later pointing $(\mathrm{P} 068+26)$ made in 2019, as seen in Fig. 1 , with a $3 \sigma$ upper limit on the flux density in Stokes $I$ of $\leq 0.39 \mathrm{mJy}$.

\section{2. $L k C a$}

During a LoTSS run on August 10, 2019, emission was found from $\mathrm{LkCa} 4$ (pointing P064+29) with a peak flux density (Stokes $I$ ) of $3.12 \pm 0.65 \mathrm{mJy}^{\text {beam }}{ }^{-1}$, as seen in Fig. 4. In Stokes $V$, the corresponding value is $1.92 \pm 0.42 \mathrm{mJy}$ beam $^{-1}$, indicating a highly circularly polarised source with a polarisation fraction of $60_{-15}^{+19} \%$. Again, as the source is unresolved, we take the peak flux density to be the total flux density.

As with KPNO-Tau 14, in order to detect changes in the emission, the direction-dependent calibrated data were divided into 30-min time bins, each of which was then imaged using WSClean in Stokes $I$ and $V$. The flux densities of several nearby compact bright sources in the field were measured in each image to check that the flux scale calibration was accurate for all of 


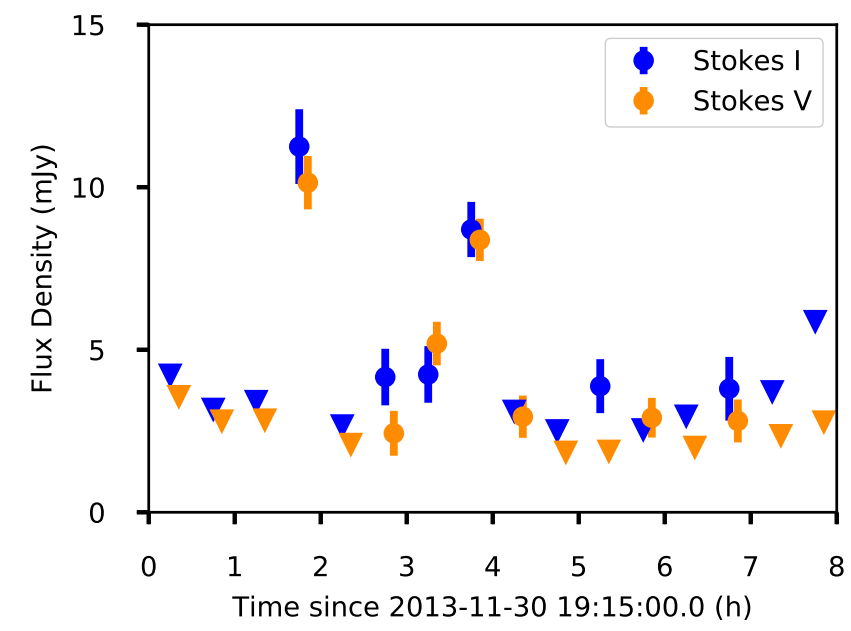

Fig. 2. Light curve of KPNO-Tau-14 over the observation with a time resolution of $30 \mathrm{~min}$. Stokes $I$ and Stokes $V$ measurements are shown by the blue and orange data points, respectively. The error bars are given by the root-mean-square noise $\sigma_{\text {rms }}$ in the image. The Stokes $V$ measurements are offset by $+0.1 \mathrm{~h}$ with respect to the Stokes $I$ measurements for better readability. We note that the absolute flux calibration error is not taken into account in the error bars as any absolute flux calibration offset should be the same for the whole observation. $3 \sigma$ upper limits are shown by the triangles for the times in which no emission was detected from the source. See Appendix D for the flux density measurements and calculated polarisation fractions.

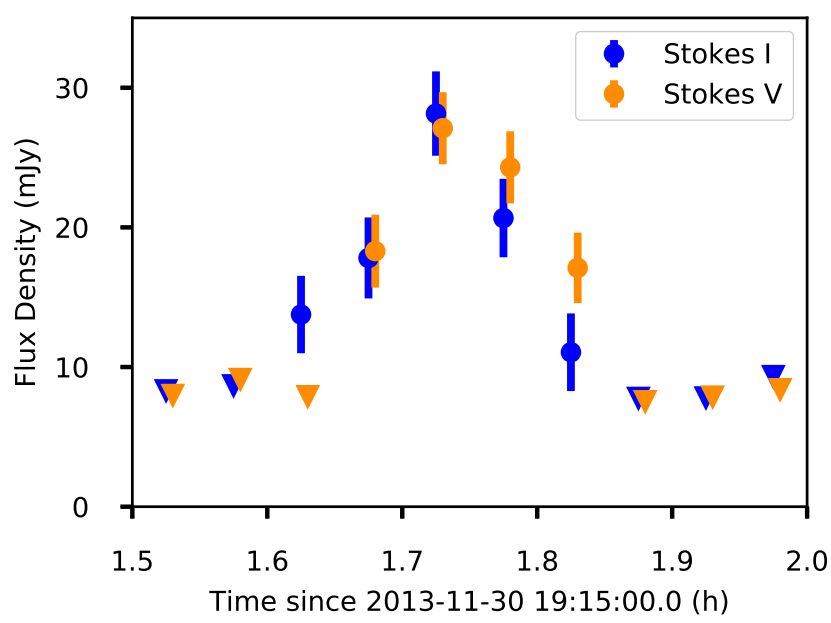

Fig. 3. Light curve of the first burst detected from KPNO-Tau-14 with a time resolution of $3 \mathrm{~min}$. Stokes $I$ and Stokes $V$ measurements are shown by the blue and orange data points, respectively. The error bars are given by the root-mean-square noise $\sigma_{\text {rms }}$ in the image. The Stokes $V$ measurements are offset by $+0.005 \mathrm{~h}$ with respect to the Stokes $I$ measurements for better readability. We note that the absolute flux calibration error is not taken into account in the error bars as any absolute flux calibration offset should be the same for the whole observation. $3 \sigma$ upper limits are shown by the triangles for the times in which no emission was detected from the source. See Appendix D for the flux density measurements and calculated polarisation fractions.

the images used. Over the $8 \mathrm{~h}$, the flux densities of these sources vary with an average standard deviation of $8 \%$ relative to the mean flux densities of each source. Since this is well within the typical absolute flux calibration error for LOFAR, we conclude that the flux density scale calibration is accurate for all of the images (see Appendix A for the light curves).
As seen from the light curve in Fig. 5, there are two bursts observed during the $8 \mathrm{~h}$ run. The first lasts from $1.0-2.5 \mathrm{~h}$ after the start of the observing period, peaking at $4.99 \pm 0.99 \mathrm{mJy}$. The second lasts from approximately $6.5-7.5 \mathrm{~h}$, peaking at $4.46 \pm$ $0.64 \mathrm{mJy}$. Both bursts are circularly polarised in a left-handed sense.

Based on the distance to the star, the peak luminosity of the first and second bursts are $\sim(1.0 \pm 0.2) \times 10^{17} \mathrm{erg} \mathrm{s}^{-1} \mathrm{~Hz}^{-1}$ and $\sim(0.9 \pm 0.1) \times 10^{17} \mathrm{erg} \mathrm{s}^{-1} \mathrm{~Hz}^{-1}$, respectively. Using a circular disk with a radius equal to the stellar radius $\sim 2.3 R_{\odot}$ (Gully-Santiago et al. 2017), as an indicative size of the source, the corresponding brightness temperatures are $\sim(1.6 \pm 0.3) \times 10^{13} \mathrm{~K}$ and $\sim(1.4 \pm$ $0.2) \times 10^{13} \mathrm{~K}$, respectively. Similar to KPNO-Tau 14 , however, it should be noted that the brightness temperatures are almost certainly much higher as the emission region is likely far smaller than the stellar disk.

We note that no emission is detected from $\mathrm{LkCa} 4$ in the 3 other relevant LoTSS pointings, P061+29, P062+26, and $\mathrm{P} 065+26$, for which the corresponding $3 \sigma$ Stokes $I$ upper limits are $\leq 0.69 \mathrm{mJy}, \leq 0.72 \mathrm{mJy}$, and $\leq 0.84 \mathrm{mJy}$, respectively.

To check if the emission is related to the rotation period of the star, the rotation phases observed at each of the observations were compared. The phases were calculated based on the barycentric Julian dates (BJD) of the observations using the following expression

$E=\frac{\mathrm{BJD}-2458662.7}{3.37}$

where $E$ is the rotational phase of the star, $3.37 \pm 0.01 \mathrm{~d}$ is the rotational period of $\mathrm{LkCa} 4$ (Grankin et al. 2008), and 2458662.7 is the BJD at the start of the first observation $(\mathrm{P} 061+29)$, which we arbitrarily chose to correspond to a rotation phase of 0.0 . The calculated rotation phases observed in each of the observations are listed in Table 3.

The two bursts observed in the LoTSS pointing P064+29 occur at phases $\approx 0.72-0.74$ and $\approx 0.79-0.80$, respectively. Therefore, the rotation phase corresponding to the first burst is observed in the observation of LoTSS pointing P065+26, which covers the phase range $0.67-0.77$. We note that there is an uncertainty in the phase of this observation relative to the observation of P064+29 of \pm 0.03 due to the uncertainty in the rotation period of the star and the period of 1 month that elapsed between the two sets of observations. Given that the source is not seen in this observation it appears that the emission is not related to the rotation phase. Although it is important to note that the two sets of observations are a month apart and the bursting mechanism may be episodic.

In addition, detecting periodicity is also made difficult by potentially variable conditions in the source region. For example, for ECM emission variable maser conditions can lead to substantial variations in flux. In the case of CR Draconis, Callingham et al. (2021) detect flux density variability of at least an order of magnitude, which is proposed to be due to 'hotspots' in the stellar magnetosphere, containing unusually dense and/or hot plasma, creating highly localised and variable emission. CM emission is generally also highly beamed, which may affect the ability to detect it. If the emission beam is frequently aligned away from our line-of-sight it will not be detected, making it difficult to identify any periodicity.

\subsection{Frequency analysis}

For the first burst from KPNO-Tau 14, the emission is sufficiently bright that we can construct a dynamic spectrum. This 

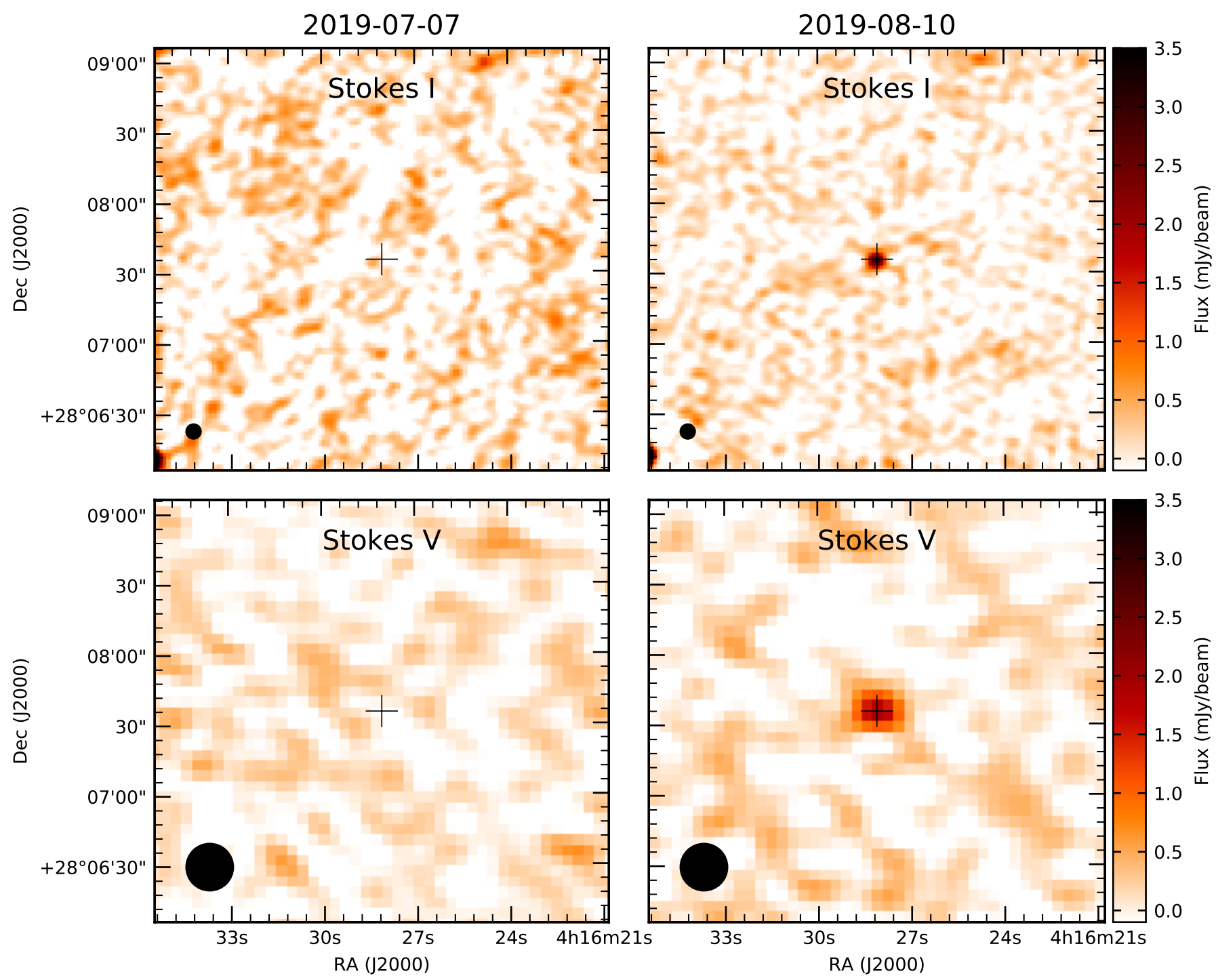

Fig. 4. Stokes $I$ (top) and Stokes $V$ (bottom) images of LkCa 4 obtained from LOFAR at two epochs: July 7, 2019 (left) and August 10, 2019 (right). The restoring beams used for the images were $6^{\prime \prime} \times 6^{\prime \prime}$ and $20^{\prime \prime} \times 20^{\prime \prime}$ for the Stokes $I$ and Stokes $V$ images, respectively. The optical position of LkCa 4 obtained from Gaia DR2 (Gaia Collaboration 2018) is indicated by the cross.

was done by binning the data for the burst according to time and frequency and then imaging the region around KPNO-Tau 14 in Stokes $I$ in each time-frequency bin and measuring the flux density of KPNO-Tau 14 in each image. The resulting dynamic spectrum is seen in Fig. 6, with a time resolution of 6 min and a frequency resolution of $18.5 \mathrm{MHz}$.

Using this dynamic spectrum, we can analyse the spectral properties of the burst and search for frequency drift. It can be seen that the burst occurs over the entire bandwidth of the observation. The emission appears to be flat over the bandwidth, with a spectral index at $1.75 \mathrm{~h}$ of $-0.03 \pm 0.61$, and the flux density seems to rise and fall similarly across the bandwidth with no frequency drift apparent. However, it should be noted that the time and frequency resolution is very low, and so we cannot rule out any finer time-frequency structure that might be present. In addition, due to the low signal-to-noise ratio and non-detections in many of the time-frequency bins, significant changes in the spectrum of the burst could be hidden by the noise.

Unfortunately, for the other radio bursts detected, the flux density is not bright enough to obtain dynamic spectra due to the low signal-to-noise ratio.

\subsection{Searching for linear polarisation}

To search for linear polarisation, rotation measure (RM) synthesis (Brentjens \& De Bruyn 2005) was used. This is required in order to correct for any Faraday rotation in the source, and/or from the foreground, an effect where the plane of linear polarisation rotates as it propagates through a magnetised plasma. The amount of rotation is frequency dependent:

$\chi(\lambda)=\chi_{0}+\phi \lambda^{2}$,

where $\chi(\lambda)$ is the observed final angle, $\chi_{0}$ is the initial angle, and $\phi$ is the Faraday depth, which depends on the average electron density and longitudinal magnetic field $\phi \propto \int n_{\mathrm{e}} \mathbf{B} \cdot \mathrm{d} \mathbf{r}$. As a result, when integrating a signal over a sufficiently wide bandwidth, the signal can become depolarised. This effect is particularly strong for LOFAR, as it operates at such long wavelengths and has a large fractional bandwidth. Rotation measure synthesis can get around this problem by imaging the $\mathrm{Q}$ and $\mathrm{U}$ images in narrow frequency channels and then performing a Fourier transform from the linearly polarised emission as a function of $\lambda^{2}$ to the linearly polarised emission as a function of $\phi$. 


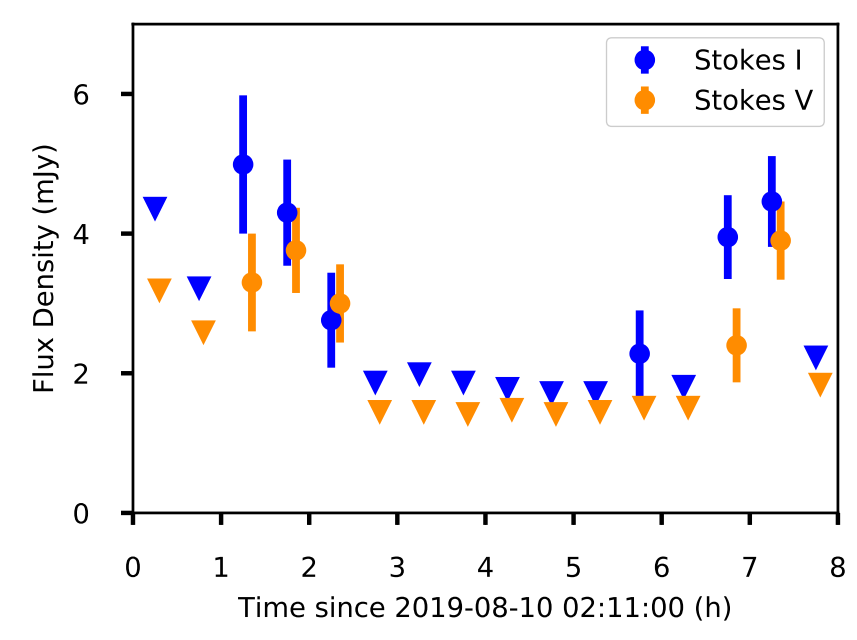

Fig. 5. Light curve of LkCa 4 over the observation with a time resolution of $30 \mathrm{~min}$. Stokes $I$ and Stokes $V$ measurements are shown by the blue and orange data points, respectively. The error bars are given by the root-mean-square noise $\sigma_{\text {rms }}$ in the image. The Stokes $V$ measurements are offset by $+0.1 \mathrm{~h}$ with respect to the Stokes $I$ measurements for better readability. We note that the absolute flux calibration error is not taken into account in the error bars as any absolute flux calibration offset should be the same for the whole observation. $3 \sigma$ upper limits are shown by the triangles for the times in which no emission was detected from the source. See Appendix D for the flux density measurements and calculated polarisation fractions.

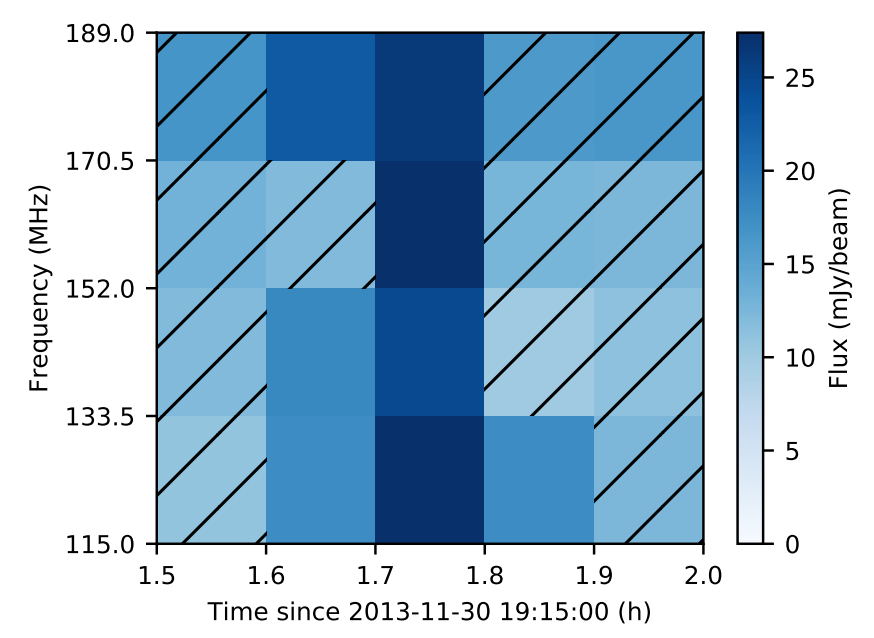

Fig. 6. Dynamic spectrum of the first burst detected from KPNO-Tau 14 The burst was divided into time-frequency bins with a time resolution of $6 \mathrm{~min}$ and a frequency resolution of $18.5 \mathrm{MHz}$. The source was then imaged in each bin and its flux density was measured. $3 \sigma$ upper limits are shown for the bins in which no emission was detected, indicated by the hatched lines. The mean noise of the images used for this spectrum is $4.4 \mathrm{mJy}$.

When RM synthesis was done at the position of KPNOTau-14, no significant linearly polarised emission was found at any Faraday depth in the Faraday dispersion spectrum. The noise level in the Faraday dispersion is $189 \mu \mathrm{Jy} \mathrm{beam}^{-1} \mathrm{RMSF}^{-1}$, where RMSF is the RM spread function, a point spread function in Faraday space due to finite sampling in $\lambda^{2}$-space. This gives a $3 \sigma$ upper limit of $<17 \%$ on the linear polarisation fraction.

Similarly for $\mathrm{LkCa} 4$, no evidence for linearly polarised emission was found. The noise level is $237 \mu \mathrm{Jy}_{\text {beam }}{ }^{-1} \mathrm{RMSF}^{-1}$, giving a $3 \sigma$ upper limit on the linear polarisation fraction of $<35 \%$.
However, this does not rule out the possibility of linear polarisation as we may be unable to detect it due to lack of sensitivity. For example, Callingham et al. (2021) detect linear polarisation from similar emission in CR Draconis with a linear polarisation fraction of $<10 \%$, which would be too faint to detect in the emission we observe.

\section{Discussion}

\subsection{Determining the relevant emission mechanism}

Both sources show a high level of circular polarisation in the bursts observed. In addition, their brightness temperatures are at least of the order of $\sim 10^{13}-10^{15} \mathrm{~K}$. This indicates a coherent emission mechanism, as incoherent mechanisms are limited to $10^{11}-10^{12} \mathrm{~K}$ since above this, the source would be rapidly cooled by inverse Compton scattering (Kellermann \& PaulinyToth 1969).

Assuming we are dealing with coherent radiation, the options are either plasma emission, as seen for example in the Sun from Type II radio bursts (Hariharan et al. 2014), or ECM emission, which is associated with auroral emitters such as planets, brown dwarfs and main sequence M dwarfs (Lynch et al. 2017; Pineda et al. 2017; Villadsen \& Hallinan 2019; Zic et al. 2019; Vedantham et al. 2020; Callingham et al. 2021) and possibly Type IV solar radio bursts (Treumann et al. 2011).

\subsection{Plasma emission}

Plasma emission occurs at the local plasma frequency, $v_{\mathrm{p}}$, and its second harmonic, but rarely if ever at higher harmonics (Dulk 1985):

$v_{\mathrm{p}}=\sqrt{n_{\mathrm{e}} e^{2} / \pi m_{\mathrm{e}}} \approx 9 \sqrt{n_{\mathrm{e}}} \mathrm{kHz}$,

where $n_{\mathrm{e}}$ is the local electron number density in $\mathrm{cm}^{-3}$. Here we assumed for simplicity that no magnetic field is present. At the fundamental frequency, plasma emission is expected to be polarised. However, the second harmonic is usually not polarised or only weakly polarised ( $<50 \%$; Melrose et al. 1978). Given the high polarisation fraction of the emission in both our sources, second harmonic plasma emission can therefore be ruled out.

The origin of plasma emission involves the production of longitudinal waves, known as Langmuir waves, in a plasma. These are usually generated by the injection of a hot plasma into a cooler ambient plasma. Some of the energy in the Langmuir waves is subsequently converted into electromagnetic waves.

Plasma emission, especially at higher frequencies, can suffer from free-free absorption due to overlying lower-density plasma blanketing the plasma in the source region (Dulk 1985). However, at low frequencies this is generally not an issue as the freefree optical depth is proportional to frequency: $\tau_{\mathrm{ff}} \propto v^{2}$.

The maximum possible brightness temperature that can be generated by plasma emission can be estimated from the effective temperature of the Langmuir waves $T_{\mathrm{L}}$, defined by (Dulk 1985):

$W_{\mathrm{L}}=\int \frac{k_{\mathrm{B}} T_{\mathrm{L}}}{(2 \pi)^{3}} \mathrm{~d}^{3} \mathbf{k}$,

where $W_{\mathrm{L}}$ is the energy density of the Langmuir waves. It has been shown that the saturated value of $W_{\mathrm{L}}$ is $10^{-5}$ times the energy density of the background plasma (i.e. $W_{\mathrm{L}} \approx 10^{-5} k_{\mathrm{B}} T$; Benz 1993), where $T$ is the temperature of the background 
plasma. From this, the limiting value of $T_{\mathrm{L}}$ can be shown to be (Dulk 1985):

$T_{\mathrm{L}} \lesssim 10^{8} \frac{v_{\mathrm{o}}^{2}}{c^{2}} \frac{v_{\mathrm{o}}}{\omega_{\mathrm{p}}} T$,

where $v_{\mathrm{o}}$ is the velocity of the fast electrons in the hot plasma component and $\omega_{\mathrm{p}}=2 \pi v_{\mathrm{p}}$. Given that the brightness temperature of the plasma emission is limited to the effective temperature of the Langmuir waves, this also gives an upper limit on the brightness temperature.

From X-ray observations (Güdel et al. 2007), the average electron temperature in the corona of KPNO-Tau 14 is known to be $1.65 \times 10^{7} \mathrm{~K}$. Using this as an estimate for $T$ and assuming a value of $v_{\mathrm{o}}$ similar to those seen in the Sun, $\approx 0.1 c-0.5 c$, the brightness temperature is therefore limited to $T_{\mathrm{b}} \lesssim 10^{15} \mathrm{~K}$. While this is just about consistent with the estimated brightness temperatures of the bursts detected, it should be noted that those values were estimated based on an emission region equal to the size of the stellar disk and so are only lower limits. In reality, the emission region is likely much smaller and therefore the brightness temperature much higher.

This suggests that plasma emission is unlikely to be able to explain the bursts observed. Additionally, if the emission was coming from the entire stellar disk, this would imply the emission was due to flaring loops occurring across the entire stellar disk. If this were the case, we would not expect to obtain such high polarisation fractions as flaring loops from regions with oppositely directed magnetic fields would generate emission of oppositely handed polarisation, which would cancel each other out. Thus a high polarisation fraction such as that observed would require a smaller emission region.

Given that the temperature of the corona used is only an average, it is possible that the temperature could be higher in smaller regions of the corona. However, this runs into the issue again that the brightness temperature would then be required to be much higher in a smaller emission region and this would in turn require an even higher plasma temperature.

For LkCa 4, unfortunately no estimates of the ambient coronal temperature are available. However, assuming it is within the range typically seen for TTSs (i.e., $10^{6}-10^{7} \mathrm{~K}$; Güdel et al. 2007), and taking the same estimates for $v_{\mathrm{o}}$ as before, the brightness temperature for plasma emission is limited to $T_{\mathrm{b}} \lesssim$ $10^{14}-10^{15} \mathrm{~K}$. Again, this is marginally consistent with the estimated brightness temperatures of the bursts. Similar to KPNOTau 14 however, these estimated values are only lower limits based on an emission region equal in size to the stellar disk. As the real emission region is likely much smaller and therefore the brightness temperature likely much higher, it seems unlikely that plasma emission can explain the bursts.

\subsection{ECM emission}

Electron-cyclotron maser emission is produced when radiation is amplified by stimulated emission at the local cyclotron frequency and its harmonics (Dulk 1985):

$v_{\mathrm{c}}=2.8 \mathrm{BMHz}$,

where $B$ is the local magnetic field strength in G. In order for ECM emission to occur, the following conditions must be met: (a) a population inversion must exist in the electron energy distribution so that amplification by stimulated emission can occur, (b) the plasma frequency must be less than the cyclotron frequency, that is, either a strong magnetic field or a low-density

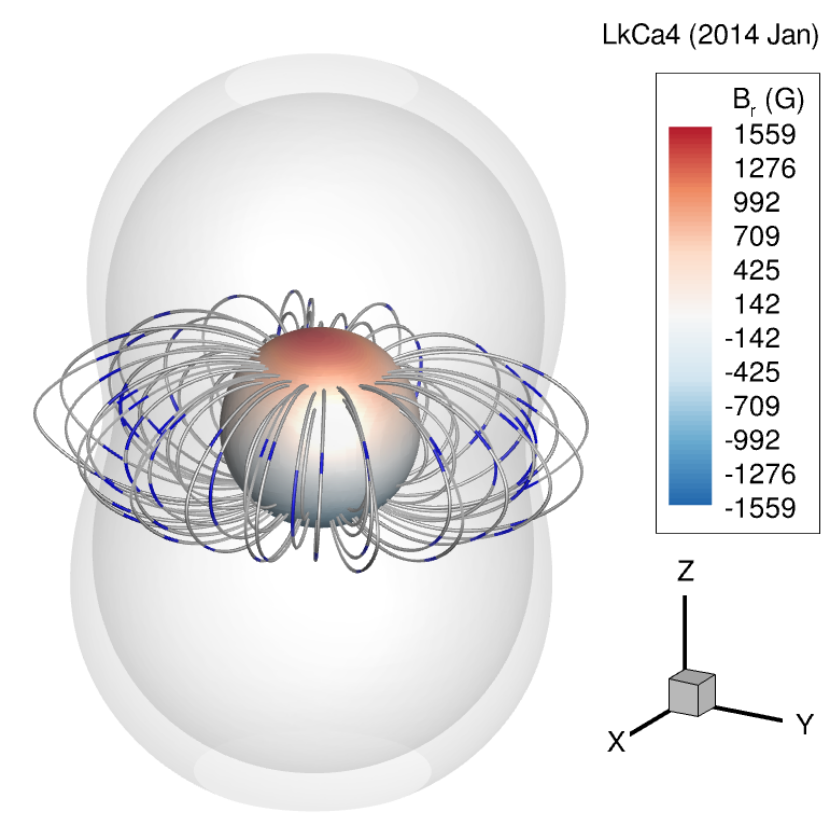

Fig. 7. 3D magnetic map of $\mathrm{LkCa} 4$, obtained by extrapolating the surface magnetic field maps obtained by Donati et al. (2014). The positive magnetic field strength values indicate where the magnetic field is pointing outwards from the surface, while the negative values indicate where it is pointing inwards towards the surface. The model used to calculate this magnetic map assumes that the field lines become fully radial at $3.4 R_{*}$. The grey surfaces indicate the region in the magnetosphere where the magnetic field strength is $40-70 \mathrm{G}$. The locations along the magnetic field lines where the magnetic field strength is in this range are indicated by the blue line segments. We note that only the closed magnetic field lines are shown in this figure.

plasma is required. We note that while the bandwidth of an individual pulse of ECM radiation is small, the frequency range of the emission as a whole can be quite large depending on the size of the emission region and the range of magnetic field strengths that it spans.

One issue for ECM emission is the absorption of the emission by overlying plasma due to gyro-resonance absorption (Dulk 1985). This is particularly an issue for fundamental ECM emission as this is expected to be strongly absorbed at the second gyro-resonance layer (where $B=B_{\mathrm{src}} / 2$ ). One way to reduce this problem is to assume the emission is produced at the second harmonic. In this case, the emission only has to cross the third harmonic layer (where $B=2 B_{\text {src }} / 3$ ). Alternatively, this issue can be avoided if the emission is generated in a low-density cavity within the magnetosphere, as this would give rise to a lower optical depth (Villadsen \& Hallinan 2019; Zic et al. 2019).

If we are dealing with ECM emission, then we can calculate the local magnetic field strength in the source region. Given the observed LOFAR frequency range, and assuming the that it is fundamental ECM emission, the emission must be from a region with a field strength ranging from $40 \mathrm{G}$ to $70 \mathrm{G}$.

For LkCa 4, Donati et al. (2014) show that the magnetosphere consists primarily of a $1.6 \mathrm{kG}$ magnetic dipole component. Assuming a dipolar magnetosphere as an approximation, the distance of the emitting region from the star can then be estimated. This gives an emission region location at a height of $\approx 2 R_{*}$. A more accurate estimate can be obtained by extrapolating the surface magnetic maps obtained by Donati et al. (2014) to obtain a 3-D map of the magnetosphere of $\mathrm{LkCa} 4$, shown in Fig. 7. The grey surfaces indicate the region in the 
magnetosphere where the magnetic field strength is $40-70 \mathrm{G}$. The locations along the closed magnetic field lines where the magnetic field strength is in this range are indicated by the blue line segments, suggesting that the emission is from a region at a height of $\approx 2 R_{*}$.

No surface field strength estimates are available for KPNOTau 14. Assuming, however, that it is of the same order of magnitude as $\mathrm{LkCa} 4$, then the emission should be from a similar height.

However, we are limited by the observation bandwidth. The frequency range of the ECM emission could extend well beyond that of LOFAR, which would imply larger emission regions and therefore a larger range of magnetic field strengths covered. For example, in the case of Earth and Jupiter, ECM emission has been observed with a frequency range of an order of magnitude or more (Melrose 2017).

Finally, it is worth repeating that for ECM emission to occur, the condition $v_{\mathrm{p}} \lesssim v_{\mathrm{c}}$ must be met (Dulk 1985). Therefore, an upper limit on the plasma density for both stars in their emitting regions can be obtained: $n_{\mathrm{e}} \lesssim 10^{8} \mathrm{~cm}^{-3}$.

\subsubsection{Coronal loops}

One method of producing ECM emission is through a flaring coronal loop in the magnetosphere of the star. Heated thermal plasma is injected into the loop and creates a loss-cone distribution through magnetic mirroring at either end (Dulk 1985).

The brightness temperature generated by such a loop would depend on its size. To determine the dimensions of the loop needed to attain the observed brightness temperatures, we use the model given by Vedantham et al. (2020), which assumes a continuously operating loss-cone maser. This model suggests coronal loops of $10^{3} R_{*}$ in the case of KPNO-Tau 14 , and $10^{2} R_{*}$ for $\mathrm{LkCa} 4$. These values are far too large to be realistic suggesting the emission cannot be generated by this mechanism.

\subsubsection{Co-rotation breakdown}

Electron-cyclotron maser emission can be produced via the breakdown of co-rotation between the plasma surrounding the star and its magnetic field. This results in field-aligned currents that accelerate electrons into the corona and chromosphere of the star, creating the population inversion needed for ECM emission. Effectively this is a scaled-up version of the mechanism driving auroral radio emission in the case of Jupiter (Zarka 1998) and ultra-cool dwarfs (Nichols et al. 2012). More recently, this mechanism has been proposed to explain the LOFAR detected main-sequence M dwarf CR Draconis (Callingham et al. 2021).

Both KPNO-Tau 14 and $\mathrm{LkCa} 4$ are fast rotators, with rotation periods of 1.86 days (Scholz et al. 2018) and 3.37 days (Grankin et al. 2008), respectively. In addition, both show evidence of intense stellar activity, given their X-ray fluxes and, in the case of $\mathrm{LkCa} 4$, high starspot coverage. Certainly, such activity could provide a source of plasma to drive the emission. In addition, WTTSs are expected to have intense ionised winds with mass-loss rates, of the order of $10^{-12}-10^{-9} M_{\odot}$ (Vidotto \& Donati 2017), which is many orders of magnitude stronger than the Solar wind. Again such winds may prove to be a source of the plasma.

In the case of ultra-cool dwarfs and Jupiter, auroral radio emission is thought to occur at a wide range of heights along magnetic field lines from near the surface to several radii of the object (Nichols et al. 2012; Zarka 1998), giving rise to a wide frequency range of emission. If this is also the case for
KPNO-Tau 14 and $\mathrm{LkCa} 4$, this would be consistent with the inferred emission region at a height of $\approx 2 R_{*}$ for $\mathrm{LkCa} 4$. One would then also expect to see ECM emission at a wide range of frequencies, above and below that observed with LOFAR. For $\mathrm{LkCa} 4$, with a measured polar magnetic field strength of $2 \mathrm{kG}$ (Donati et al. 2014), this would imply ECM emission could, in principle, be detected at frequencies of up to $5.6 \mathrm{GHz}$ (but see Sect. 5).

\subsubsection{Star-planet interaction}

Another possible method for generating ECM emission is through the interaction of a stellar wind with the magnetosphere of an orbiting exoplanet. This is analogous to what happens in the Solar System with the giant planets (Farrell et al. 1999). For example Vidotto et al. (2010) and Lynch et al. (2018) predict that hot Jupiters orbiting young stars should produce the highest flux density of radio emission of any type of exoplanet.

From magnetohydrodynamical simulations of the stellar wind of the host star, Vidotto \& Donati (2017) predict that the exoplanet V830 Tau b, a 2 Myr-old hot Jupiter orbiting a WTTS at $0.057 \mathrm{au}$, should produce an average radio flux density of 6-24 mJy, for a planet with a radius of 1-2 Jupiter radii. However, the flux density would not be constant, as it would vary over the planet's orbit as it moves through different regions of the stellar wind. This radio emission would be emitted at frequencies between $28 \mathrm{MHz}$ and $240 \mathrm{MHz}$, depending on the planetary magnetic field strength $(10-100 \mathrm{G})$, meaning that such emission could potentially be detectable with LOFAR.

Given that V830 Tau is a WTTS, like KPNO-Tau 14 and $\mathrm{LkCa} 4$, and is at a similar distance (147 pc), a hot Jupiter orbiting these sources could be able to generate similar levels of radio emission. In order for such emission to be observed using LOFAR at HBA frequencies, the surface magnetic field strength of the planet would need to be $\gtrsim 50 \mathrm{G}$.

Alternatively, another method of generating radio emission through star-planet interaction is through a sub-Alfvénic interaction, similar to that seen in the Jupiter-Io interaction, which causes the Io-dependent decametric (Io-DAM) radio emission, in which the stellar magnetosphere couples with an orbiting body (Saur et al. 2013; Turnpenney et al. 2018). Magnetic energy flux is carried by Alfvén waves from the orbiting exoplanet and back towards the star, generating radio emission along the field line connecting the star and the planet. This was recently proposed to cause the radio emission seen with LOFAR in the nearby $M$ dwarf GJ 1151 (Vedantham et al. 2020), and later confirmed through detection of a compatible exoplanet using the radial velocity method (Mahadevan et al. 2021).

To check if this is a plausible explanation for the emission that we have detected, we calculated the theoretical estimate for the power of the Alfvén waves generated by the interaction based on the following expression (Zarka 2007; Saur et al. 2013):

$P_{\mathrm{th}}=\frac{1}{2} R^{2} v_{\mathrm{rel}}(B \sin \theta)^{2} M_{\mathrm{A}}$,

where $R$ is the radius of the obstacle to the plasma flow (i.e. the planetary magnetosphere), $v_{\text {rel }}$ is the relative velocity of the stellar wind with respect to the planet, $B$ is the stellar magnetic field strength at the location of the planet, $\theta$ is the angle between the stellar magnetic field and $v_{\text {rel }}$, and $M_{\mathrm{A}}$ is the Alfvén Mach number at the position of the planet. Details of the model of the stellar wind and magnetic field used are given in Appendix C. 
This was then compared with the observationally implied power based on the flux density observed:

$P_{\text {obs }}=F \Omega d^{2} \Delta v \epsilon$,

where $F$ is the observed radio flux density, $\Omega$ is the solid angle into which the emission is beamed, $d$ is the distance to the source, $\Delta v$ is the total bandwidth of the emission, and $\epsilon$ is the efficiency of conversion of the energy of the Alfvén waves to radio emission. The solid angle was estimated to be $\Omega=1.6 \mathrm{sr}$, similar to that of the Jovian radio emission (Zarka et al. 2004). The total bandwidth was taken to be the peak cyclotron frequency of the star $\Delta v \approx 2.8 B_{0} \mathrm{MHz}$, where $B_{0}$ is the surface magnetic field strength of the star in $\mathrm{G}$.

For $\mathrm{LkCa} 4$, again taking a surface magnetic field strength of $1.6 \mathrm{kG}$, this gives $P_{\mathrm{obs}} \sim 6 \times 10^{27} \mathrm{erg} \mathrm{s}^{-1}$ for the brightest burst observed. No magnetic field strength measurements are available for KPNO-Tau 14. Therefore, we used an estimate of $1 \mathrm{kG}$, which gives $P_{\mathrm{obs}} \sim(2.8 \pm 0.4) \times 10^{28} \mathrm{erg} \mathrm{s}^{-1}$ for the brightest burst observed. The efficiency of conversion was taken to be $\epsilon=0.01$, similar to the value seen for the Io-DAM emission.

If, for example, there were a planet orbiting at $10 R_{*}$, it could be shown by using Eqs. (7) and (8) that such values are appropriate to a planet with a magnetosphere of radius $0.6 R_{\mathrm{J}}$, where $R_{\mathrm{J}}$ is the radius of Jupiter, for $\mathrm{LkCa} 4$, or a planet with a magnetosphere of radius $2 R_{\mathrm{J}}$ for KPNO-Tau 14 . Such planets would likely be hot Jupiters. However, due to the many unknown parameters in this calculation, for example the solid angle of the emission, the efficiency of conversion, the orbital semi-major axis of the planet, these are only very rough estimates.

Based on these calculations, it seems that a star-planet interaction is at least a plausible explanation for the radio emission observed, either due to a stellar wind interaction or a sub-Alfvénic interaction. In order to determine whether or not the emission is of planetary origin, the YSOs would have to be monitored for a longer time to see if there is any periodicity in the radio emission unrelated to the rotation period of the star. A detection of an exoplanet around either source orbiting close to the star would also be a strong piece of evidence in favour of this. Unfortunately, detecting exoplanets in orbit around TTSs is difficult. Due to their high levels of activity, they generate large fluctuations in rotational velocity (RV). This makes it difficult to detect any orbiting exoplanets, even hot Jupiters.

Neither of the two sources is known to host any exoplanets. In the case of LkCa 4, Donati et al. (2014) attempted to detect a hot Jupiter by searching for periodic signals in its RV curve, after filtering out the RV variations due to the activity of the star. However, they were unable to find any signal with a semi-amplitude greater than $0.1 \mathrm{~km} \mathrm{~s}^{-1}$, and conclude that there is no evidence for a hot Jupiter around $\mathrm{LkCa}$, although they suggest that it is possible that there could be a planet with a similar orbital period to the rotation period of the star as its RV signal would then be hidden.

\section{Detecting ECM emission in higher-frequency radio emission from YSOs}

As stated earlier, until the availability of facilities such as LOFAR and the GMRT, most radio observations of YSOs have been made at centimetre wavelengths (Anglada et al. 2018). In these cases, what is observed on source tends to fall into two categories: either relatively steady (over at least several months) thermal bremsstrahlung emission arising from the ionised component of an outflow (Ray \& Ferreira 2021) or non-thermal flarelike emission, sometimes with a quasi-continuous background, that can vary over periods even less than an hour (Forbrich et al. 2017). The source of this emission is usually attributed to gyrosynchrotron radiation, produced by mildly relativistic electrons with energies around a few $\mathrm{MeV}$. While thermal emission from winds or outflows has only been seen from CTTSs (or their embedded counterparts) in the solar-mass regime, non-thermal emission is mainly observed from weak-line T Tauri systems, although it has also been seen in less evolved systems (Dzib et al. 2015). In all of these stars high energy processes are believed to be operating, however, in the case of less evolved systems, the non-thermal emission is expected to be absorbed by an overlying plasma (Andre 1996).

As ECM emission has been detected here at metre wavelengths from two YSOs, it is reasonable to ask whether some, or all, of the non-thermal radio flux in the centimetre band seen in other YSOs could be attributed to the same mechanism. The first point to note is that, if ECM emission is present, we expect to find a very high level of circular polarisation $(\approx 100 \%)$. In addition we would also anticipate very high surface brightness temperature measurements. Only a few detections of circular polarisation from YSOs at centimetre wavelengths exist and certainly some (Skinner 1993; Feigelson et al. 1998) appear consistent with optically thin gyro-synchrotron emission (Dulk 1985) where the circular polarisation fraction can reach at most a few tens of a per cent. That this is the appropriate mechanism is then backed up by the expected surface brightness temperature measurements. We are aware of just one YSO, T Tau Sb, where approximately $100 \%$ circular polarisation was observed on source at centimetre wavelengths (Smith et al. 2003) and another, EM* SR 20, in the decimetre regime (Pritchard et al. 2021). In at least the former case, the measured size of the emitting region, in combination with the flux, also suggests a coherent mechanism must be responsible. Further observations are clearly required to ascertain whether the type of emission observed here is common amongst YSOs at low frequencies or whether it is the rarity seen at higher frequencies.

\section{Conclusions}

We detect flaring radio emission from two WTTSs, KPNOTau 14 and LkCa 4, located in the TMC, at $\sim 150 \mathrm{MHz}$ using LOFAR. The emission detected from both sources is strongly circularly polarised and highly variable over the course of each observation.

Based on the characteristics of the emission, in particular the high brightness temperature $\left(\gtrsim 10^{13}-10^{15} \mathrm{~K}\right)$ and polarisation fraction (60-90\%), we conclude that the emission must be due to a coherent emission mechanism, either plasma or ECM emission. Of the two coherent emission mechanisms, plasma emission seems unlikely as it cannot generate the high brightness temperatures and polarisation fractions observed, and so we conclude that ECM must be responsible.

In order to generate ECM emission, a breakdown of corotation between plasma surrounding the star and the stellar magnetosphere is the most likely explanation. Given that WTTSs are expected to have high mass-loss rates, this would provide a source of plasma to drive the emission. Such emission would be a scaled-up version of the auroral radio emission seen in Jupiter and ultra-cool dwarfs.

Alternatively, ECM emission could also be generated through a star-planet interaction. This could either be an interaction of the stellar wind with the planetary magnetosphere of an orbiting exoplanet, as seen in our own Solar System with the giant planets, or a sub-Alfvénic interaction of the stellar 
magnetosphere with the exoplanet, similar to the Jupiter-Io interaction and seen recently in the M dwarf GJ 1151. If a star-planet interaction is responsible, it could provide important information about exoplanetary magnetospheres. Additionally, given the difficulty in detecting exoplanets around YSOs using traditional methods, this could provide an alternative way of detecting young exoplanets.

These detections suggest that low-frequency radio observations are a valuable method for studying YSOs. As mentioned earlier, the Taurus and Perseus molecular clouds have recently been surveyed using LOFAR. Therefore, we may potentially detect similar emission from other YSOs in these clouds as part of this survey and other observations at low frequencies, giving us important information about their magnetic activity and potentially indicating the presence of exoplanets.

Acknowledgements. A.F.J., S.J.D.P. and T.P.R. acknowledge funding from the European Research Council (ERC) under Advanced Grant No. 743029. A.A.V acknowledges funding from the European Research Council (ERC) under the European Union's Horizon 2020 research and innovation programme (grant agreement No. 817540, ASTROFLOW). This paper is based on data obtained with the International LOFAR Telescope (ILT) under project codes LC1_001 and LC12_015. LOFAR (Van Haarlem et al. 2013) is the Low Frequency Array designed and constructed by ASTRON. It has observing, data processing, and data storage facilities in several countries, that are owned by various parties (each with their own funding sources), and that are collectively operated by the ILT foundation under a joint scientific policy. The ILT resources have benefitted from the following recent major funding sources: CNRS-INSU, Observatoire de Paris and Université d'Orléans, France; BMBF, MIWF-NRW, MPG Germany; Science Foundation Ireland (SFI), Department of Business, Enterprise and Innovation (DBEI), Ireland; NWO, The Netherlands; The Science and Technology Facilities Council, UK. This research made use of the Dutch national e-infrastructure with support of the SURF Cooperative (e-infra 180169) and the LOFAR e-infra group. The Jülich LOFAR Long Term Archive and the German LOFAR network are both coordinated and operated by the Jülich Supercomputing Centre (JSC), and computing resources on the supercomputer JUWELS at JSC were provided by the Gauss Centre for Supercomputing e.V. (grant CHTB00) through the John von Neumann Institute for Computing (NIC). This research made use of the University of Hertfordshire high-performance computing facility and the LOFAR-UK computing facility located at the University of Hertfordshire and supported by STFC [ST/P000096/1], and of the Italian LOFAR IT computing infrastructure supported and operated by INAF, and by the Physics Department of Turin university (under an agreement with Consorzio Interuniversitario per la Fisica Spaziale) at the C3S Supercomputing Centre, Italy. This research made use of Matplotlib (Hunter 2007), Scipy (Virtanen et al. 2020), and of APLpy, an open-source plotting package for Python (Robitaille \& Bressert 2012).

\section{References}

Ainsworth, R. E., Scaife, A. M. M., Ray, T. P., et al. 2014, ApJ, 792, L18 Andre, P. 1996, in Radio Emission from the Stars and the Sun, eds. A. R. Taylor, \& J. M. Paredes, ASP Conf. Ser., 93, 273

Anglada, G., Rodríguez, L. F., \& Carrasco-González, C. 2018, A\&ARv, 26, 3

Bailer-Jones, C. A. L., Rybizki, J., Fouesneau, M., Mantelet, G., \& Andrae, R. 2018, AJ, 156, 58

Baraffe, I., Homeier, D., Allard, F., \& Chabrier, G. 2015, A\&A, 577, A42

Benz, A. O. 1993, Plasma Astrophysics: Kinetic Processes in Solar and Stellar Coronae, 184 (Dordrecht: Springer)

Brentjens, M. A., \& De Bruyn, A. G. 2005, A\&A, 441, 1217

Callingham, J. R., Pope, B. J. S., Feinstein, A. D., et al. 2021, A\&A, 648, A13

Condon, J. J., Cotton, W. D., Greisen, E. W., et al. 1998, AJ, 115, 1693

de Gasperin, F., Dijkema, T. J., Drabent, A., et al. 2019, A\&A, 622, A5

Donati, J. F., Hébrard, E., Hussain, G., et al. 2014, MNRAS, 444, 3220

Dulk, G. A. 1985, ARA\&A, 23, 169

Dulk, G. A., Leblanc, Y., \& Lecacheux, A. 1994, A\&A, 286, 683

Dzib, S. A., Loinard, L., Rodríguez, L. F., et al. 2015, ApJ, 801, 91

Esplin, T. L., Luhman, K. L., \& Mamajek, E. E. 2014, ApJ, 784, 126

Farrell, W. M., Desch, M. D., \& Zarka, P. 1999, J. Geophys. Res., 104, 14025

Feeney-Johansson, A., Purser, S. J. D., Ray, T. P., et al. 2019, ApJ, 885, L7

Feigelson, E. D., \& Montmerle, T. 1999, ARA\&A, 37, 363

Feigelson, E. D., Carkner, L., \& Wilking, B. A. 1998, ApJ, 494, L215
Fernández, M., Stelzer, B., Henden, A., et al. 2004, A\&A, 427, 263 Forbrich, J., Reid, M. J., Menten, K. M., et al. 2017, ApJ, 844, 109 Gaia Collaboration (Brown, A. G. A., et al.) 2018, A\&A, 616, A1 Grankin, K. N., Bouvier, J., Herbst, W., \& Melnikov, S. Y. 2008, A\&A, 479, 827 Güdel, M., Briggs, K. R., Arzner, K., et al. 2007, A\&A, 468, 353 Gully-Santiago, M. A., Herczeg, G. J., Czekala, I., et al. 2017, ApJ, 836, 200 Hales, S. E. G., Baldwin, J. E., \& Warner, P. J. 1988, MNRAS, 234, 919 Hardcastle, M. J., Shimwell, T. W., Tasse, C., et al. 2021, A\&A, 648, A10

Hariharan, K., Ramesh, R., Kishore, P., Kathiravan, C., \& Gopalswamy, N. 2014, ApJ, 795, 14

Hunter, J. D. 2007, Comput. Sci. Eng., 9, 90

Intema, H. T., Jagannathan, P., Mooley, K. P., \& Frail, D. A. 2017, A\&A, 598, A78

Kavanagh, R. D., Vidotto, A. A., Klein, B., et al. 2021, MNRAS, 504, 1511

Kellermann, K. I., \& Pauliny-Toth, I. I. K. 1969, ApJ, 155, L71

Kraus, A. L., White, R. J., \& Hillenbrand, L. A. 2006, ApJ, 649, 306

Luhman, K. L., Briceno, C., Stauffer, J. R., et al. 2003, ApJ, 590, 348

Lynch, C. R., Lenc, E., Kaplan, D. L., Murphy, T., \& Anderson, G. E. 2017, ApJ, 836, L30

Lynch, C. R., Murphy, T., Lenc, E., \& Kaplan, D. L. 2018, MNRAS, 478, 1763

Mahadevan, S., Stefánsson, G., Robertson, P., et al. 2021, ApJ, submitted [arXiv:2102.02233]

Melrose, D. B. 2017, Rev. Mod. Plasma Phys., 1, 5

Melrose, D. B., Dulk, G. A., \& Smerd, S. F. 1978, A\&A, 66, 315

Mohan, N., \& Rafferty, D. 2015, PyBDSF: Python Blob Detection and Source Finder, Astrophysics Source Code Library, [record ascl:1502 .007]

Mohanty, S., Jayawardhana, R., \& Basri, G. 2005, ApJ, 626, 498

Muzerolle, J., Luhman, K. L., Briceno, C., Hartmann, L., \& Calvet, N. 2005, ApJ, 625, 906

Nichols, J. D., Burleigh, M. R., Casewell, S. L., et al. 2012, ApJ, 760, 59

Offringa, A. R., McKinley, B., Hurley-Walker, N., et al. 2014, MNRAS, 444, 606

Parker, E. N. 1958, ApJ, 128, 664

Pineda, J. S., Hallinan, G., \& Kao, M. M. 2017, ApJ, 846, 75

Pritchard, J., Murphy, T., Zic, A., et al. 2021, MNRAS, 502, 5438

Ray, T. P., \& Ferreira, J. 2021, New Astron. Rev., 93, 101615

Rebull, L. M., Padgett, D. L., McCabe, C.-E., et al. 2010, ApJS, 186, 259

Robishaw, T., \& Heiles, C. 2018, ArXiv e-prints [arXiv:1806.07391]

Robitaille, T., \& Bressert, E. 2012, APLpy: Astronomical Plotting Library in Python, Astrophysics Source Code Library, [record ascl:1208.017]

Saur, J., Grambusch, T., Duling, S., Neubauer, F. M., \& Simon, S. 2013, A\&A, 552, A119

Schnepf, N. R., Lovelace, R. V. E., Romanova, M. M., \& Airapetian, V. S. 2015, MNRAS, 448, 1628

Scholz, A., Moore, K., Jayawardhana, R., et al. 2018, ApJ, 859, 153

Shimwell, T. W., Röttgering, H. J. A., Best, P. N., et al. 2017, A\&A, 598, A104

Shimwell, T. W., Tasse, C., Hardcastle, M. J., et al. 2019, A\&A, 622, A1

Skinner, S. L. 1993, ApJ, 408, 660

Smirnov, O. M., \& Tasse, C. 2015, MNRAS, 449, 2668

Smith, K., Pestalozzi, M., Güdel, M., Conway, J., \& Benz, A. O. 2003, A\&A, 406, 957

Stelzer, B., Flaccomio, E., Briggs, K., et al. 2007, A\&A, 468, 463

Tasse, C., Hugo, B., Mirmont, M., et al. 2018, A\&A, 611, A87

Tasse, C., Shimwell, T., Hardcastle, M. J., et al. 2021, A\&A, 648, A1

Treumann, R. A., Nakamura, R., \& Baumjohann, W. 2011, Ann. Geophys., 29, 1673

Turnpenney, S., Nichols, J. D., Wynn, G. A., \& Burleigh, M. R. 2018, ApJ, 854

Uzawa, A., Tsuboi, Y., Morii, M., et al. 2011, PASJ, 63, S713

Van Haarlem, M. P., Wise, M. W., Gunst, A. W., et al. 2013, A\&A, 556, A2

Van Weeren, R. J., Williams, W. L., Hardcastle, M. J., et al. 2016, ApJS, 223

Vedantham, H. K., Callingham, J. R., Shimwell, T. W., et al. 2020, Nat. Astron., 1

Vidotto, A. A., \& Donati, J. F. 2017, A\&A, 602, A39

Vidotto, A. A., Opher, M., Jatenco-Pereira, V., \& Gombosi, T. I. 2010, ApJ, 720, 1262

Villadsen, J., \& Hallinan, G. 2019, ApJ, 871, 214

Virtanen, P., Gommers, R., Oliphant, T. E., et al. 2020, Nat. Methods, 17, 261

Waterfall, C. O. G., Browning, P. K., Fuller, G. A., \& Gordovskyy, M. 2019, MNRAS, 483, 917

Yang, H., Herczeg, G. J., Linsky, J. L., et al. 2012, ApJ, 744, 121

Young, K. E., Young, C. H., Lai, S. P., Dunham, M. M., \& II, N. J. E. 2015, AJ, 150,40

Zarka, P. 1998, J. Geophys. Res., 103, 20159

Zarka, P. 2007, Planet. Space Sci., 55, 598

Zarka, P., Cecconi, B., \& Kurth, W. S. 2004, J. Geophys. Res.: Space Phys., 109, A09S 15

Zic, A., Stewart, A., Lenc, E., et al. 2019, MNRAS, 488, 559 


\section{Appendix A: Light curves of background field sources}

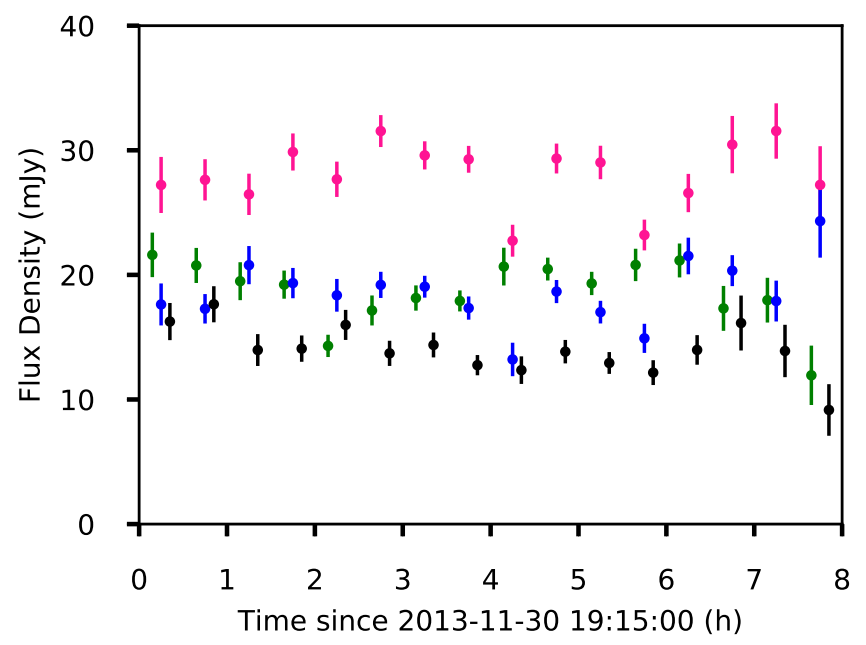

Fig. A.1. Light curves of the sources in the field of KPNO-Tau 14: J043314+261818 (pink), J043316+261813 (blue), J043349+260753 (green), and J043331+260704 (black). We note that the fluxes of $\mathrm{J} 043349+260753$ and $\mathrm{J} 043331+260704$ are offset by $-0.1 \mathrm{~h}$ and +0.1 $\mathrm{h}$, respectively, for readability. The error bars are given by the fitting error $\sigma_{\text {fit }}$ given by PyBDSF and the root-mean-square noise $\sigma_{\text {rms }}$ added in quadrature: $\sigma=\sqrt{\sigma_{\text {fit }}^{2}+\sigma_{\text {rms }}^{2}}$.

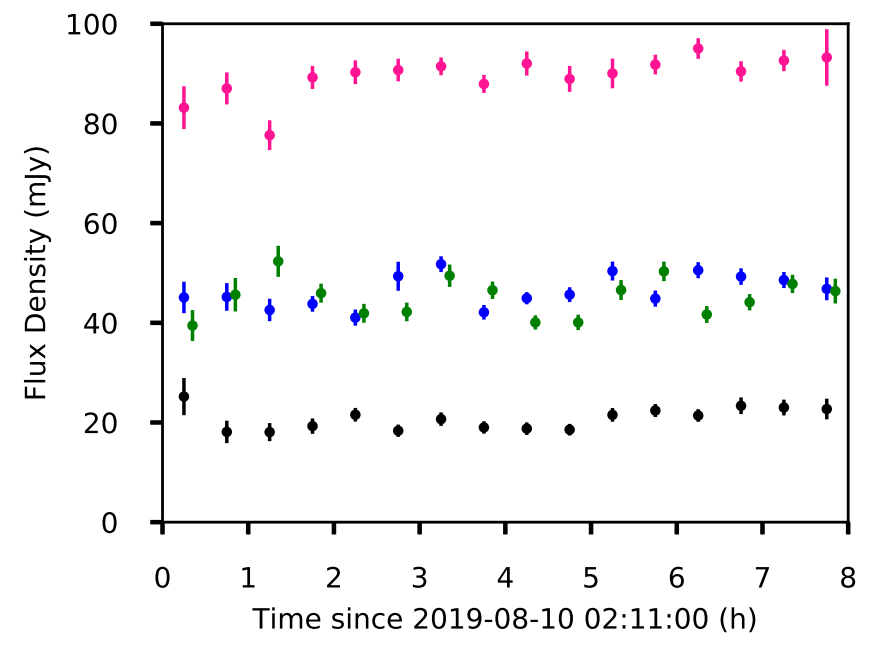

Fig. A.2. Light curves of the sources in the field of $\mathrm{LkCa} 4$ : J041556+281645 (pink), J041732+281048 (blue), J041640+280521 (green), and J041607+281833 (black). We note that the fluxes of $\mathrm{J} 041640+280521$ are offset by $+0.1 \mathrm{~h}$ for readability. The error bars are given by the fitting error $\sigma_{\text {fit }}$ given by PyBDSF and the root-meansquare noise $\sigma_{\text {rms }}$ added in quadrature: $\sigma=\sqrt{\sigma_{\text {fit }}^{2}+\sigma_{\text {rms }}^{2}}$.

\section{Appendix B: Sign of Stokes V}

There are multiple conventions for defining the Stokes parameters within radio astronomy (Robishaw \& Heiles 2018). The one used by CASA and WSClean is $V=(R R-L L) / 2$. This is different from the official IAU definition, which does not contain the factor of 2: $V=R R-L L$. Within pulsar astronomy and optical astronomy, the adopted convention is $V=L L-R R$. There are also other factors that can affect the sign of Stokes V, such as the location of the feed in the antenna (primary, secondary, tertiary focus). Therefore, it is necessary to check by comparing to sources with known circular polarisations (e.g. pulsars). This was done by Callingham et al. (in prep.) for LoTSS data, who found that the LoTSS data followed the pulsar convention (i.e. $L L-R R)$. Therefore, we can determine that the KPNO-Tau 14 and $\mathrm{LkCa} 4$ emission is left-handed.

\section{Appendix C: Stellar wind and magnetic field model}

When calculating the theoretical power of the for Alfvén waves generated by the sub-Alfvénic interaction of the stellar magnetosphere with an orbiting exoplanet, the stellar wind is assumed to have the form of a Parker spiral (Parker 1958), which is given by the solution to the equation:

$\frac{u_{r}(r)^{2}}{c_{s}^{2}}-\ln \left(\frac{u_{r}(r)^{2}}{c_{s}^{2}}\right)=4 \ln \left(\frac{r}{r_{c}}\right)+4\left(\frac{r_{c}}{r}\right)-3$,

where $u_{r}(r)$ is the radial stellar wind velocity at the radial distance $r$ from the surface of the star. The equation is parameterised by the sound speed:

$c_{s}=\sqrt{\frac{k_{\mathrm{B}} T}{m_{\mathrm{p}}}}$,

where $k_{\mathrm{B}}$ is the Boltzmann constant, $T$ is the temperature of the stellar wind, and $m_{p}$ is the proton mass. The wind passes through $c_{s}$ at the critical distance $r_{c}$ :

$r_{c}=\frac{m_{\mathrm{p}} G M_{*}}{4 k_{\mathrm{B}} T}$

where $G$ is the gravitational constant and $M_{*}$ is the mass of the star.

The magnetic field components are given by:

$B_{r}=B_{0}\left(\frac{r_{0}}{r}\right)^{2}$,

$B_{\varphi}=B_{r} \frac{u_{\varphi}-\Omega_{*} r}{v_{\mathrm{rel}}}$

where $\Omega_{*}$ is the stellar RV and $u_{\varphi}$ is the azimuthal stellar wind velocity given by $u_{\varphi}(r)=\left(\Omega_{*} R_{*}^{2}\right) / r$. 


\section{Appendix D: Flux density measurements}

Table D.1. Flux densities of KPNO-Tau 14 in Stokes I and V that were used in the light curve in Fig. 2.

\begin{tabular}{|c|c|c|c|c|}
\hline $\begin{array}{l}\text { Time } \\
\text { (hrs) }\end{array}$ & $\begin{array}{c}\text { Stokes I } \\
(\mathrm{mJy})\end{array}$ & $\begin{array}{c}\text { Stokes V } \\
(\mathrm{mJy})\end{array}$ & $\begin{array}{l}\text { Pol. Fraction } \\
(\%)\end{array}$ & Beam \\
\hline $0-0.5$ & $\leq 4.26$ & $\leq 3.60$ & - & $20^{\prime \prime} .3 \times 3^{\prime \prime} .2,-48^{\circ}$ \\
\hline $0.5-1.0$ & $\leq 3.21$ & $\leq 2.85$ & - & $18^{\prime \prime} .7 \times 3^{\prime \prime} .1,-52^{\circ}$ \\
\hline $1.0-1.5$ & $\leq 3.44$ & $\leq 2.88$ & - & $16^{\prime \prime} 6 \times 3^{\prime \prime} .1,-57^{\circ}$ \\
\hline $1.5-2.0$ & $11 . \overline{25} \pm 1.15$ & $10 . \overline{1} 4 \pm 0.82$ & $88_{-9}^{+8}$ & $15^{\prime \prime} 0 \times 3^{\prime \prime} .2,-62^{\circ}$ \\
\hline $2.0-2.5$ & $\leq 2.70$ & $\leq 2.13$ & -9 & $15^{\prime \prime} 0 \times 2^{\prime \prime} 9,-68^{\circ}$ \\
\hline $2.5-3.0$ & $4 . \overline{16} \pm 0.87$ & $2 . \overline{43} \pm 0.69$ & $57_{-18}^{+20}$ & $13^{\prime \prime} .8 \times 3^{\prime \prime} .1,-73^{\circ}$ \\
\hline $3.0-3.5$ & $4.24 \pm 0.87$ & $5.19 \pm 0.67$ & $91_{-10}^{+7}$ & $13^{\prime \prime} 0 \times 3 " .4,-82^{\circ}$ \\
\hline $3.5-4.0$ & $8.70 \pm 0.85$ & $8.38 \pm 0.65$ & $91_{-8}^{+6}$ & $14^{\prime \prime} .2 \times 4^{\prime \prime} .1,83^{\circ}$ \\
\hline $4.0-4.5$ & $\leq 3.15$ & $2.94 \pm 0.65$ & $\geq 93$ & $20^{\prime \prime} .4 \times 4^{\prime \prime} .2,66^{\circ}$ \\
\hline $4.5-5.0$ & $\leq 2.55$ & $\leq 1.89$ & - & $13^{\prime \prime} .4 \times 4^{\prime \prime} .2,62^{\circ}$ \\
\hline $5.0-5.5$ & $\leq 2.55$ & $\leq 1.89$ & - & $7 \prime .4 \times 4^{\prime \prime} .2,61^{\circ}$ \\
\hline $5.5-6.0$ & $3.88 \pm 0.83$ & $\leq 1.92$ & $\leq 49$ & $6^{\prime \prime} .2 \times 4^{\prime \prime} .2,60^{\circ}$ \\
\hline $6.0-6.5$ & $\leq 2.58$ & $2 . \overline{1} \pm 0.61$ & $\geq 112$ & $5^{\prime \prime} .7 \times 4^{\prime \prime} .3,60^{\circ}$ \\
\hline $6.5-7.0$ & $3.80 \pm 0.98$ & $2.81 \pm 0.66$ & $69_{-20}^{+18}$ & $5^{\prime \prime} .5 \times 4^{\prime \prime} .3,61^{\circ}$ \\
\hline $7.0-7.5$ & $\leq 3.75$ & $\leq 2.40$ & -20 & $5^{\prime \prime} .5 \times 4^{\prime \prime} .3,62^{\circ}$ \\
\hline $7.5-8.0$ & $\leq 5.91$ & $\leq 2.82$ & - & $5^{\prime \prime} 8 \times 4^{\prime \prime} .3,62^{\circ}$ \\
\hline
\end{tabular}

Notes. The time in the first column refers to the time in hours after the start of the observation (November 30, 2013, at 19:15:00). The polarisation fraction is shown for the times where both Stokes I and V are detected. When only Stokes I or V are detected, $3 \sigma$ upper or lower limits, respectively, are given for the polarisation fraction. The dimensions and position angle of the synthesised beam in the image at each time are shown in the final column.

Table D.2. Flux densities of KPNO-Tau 14 in Stokes I and V that were used in the light curve of the first burst in Fig. 3.

\begin{tabular}{|c|c|c|c|c|}
\hline $\begin{array}{l}\text { Time } \\
\text { (hrs) }\end{array}$ & $\begin{array}{l}\text { Stokes I } \\
(\mathrm{mJy})\end{array}$ & $\begin{array}{c}\text { Stokes V } \\
(\mathrm{mJy})\end{array}$ & $\begin{array}{l}\text { Pol. Fraction } \\
(\%)\end{array}$ & Beam \\
\hline $1.50-1.55$ & $\leq 8.40$ & $\leq 8.07$ & - & $18^{\prime \prime} .3 \times 3^{\prime \prime} .0,-60^{\circ}$ \\
\hline $1.55-1.60$ & $\leq 8.76$ & $\leq 9.21$ & - & $18^{\prime \prime} .5 \times 3^{\prime \prime} .0,-60^{\circ}$ \\
\hline $1.60-1.65$ & $13 . \overline{7} 6 \pm 2.77$ & $\leq 7.98$ & $\leq 58$ & $18^{\prime \prime} .2 \times 3^{\prime \prime} 0,-61^{\circ}$ \\
\hline $1.65-1.70$ & $17.81 \pm 2.90$ & $18 . \overline{30} \pm 2.61$ & $88_{-13}^{+8}$ & $18^{\prime \prime} .1 \times 3^{\prime \prime} .0,-61^{\circ}$ \\
\hline $1.70-1.75$ & $28.15 \pm 3.02$ & $27.10 \pm 2.58$ & $90_{-8}^{+7}$ & $18^{\prime \prime} 0 \times 3^{\prime \prime} .0,-62^{\circ}$ \\
\hline $1.75-1.80$ & $20.67 \pm 2.81$ & $24.30 \pm 2.58$ & $93_{-8}^{+5}$ & $17^{\prime \prime} .9 \times 3^{\prime \prime} .0,-62^{\circ}$ \\
\hline $1.80-1.85$ & $11.06 \pm 2.78$ & $17.10 \pm 2.52$ & $92_{-10}^{+6}$ & $17^{\prime \prime} .9 \times 3^{\prime \prime} .0,-63^{\circ}$ \\
\hline $1.85-1.90$ & $\leq 7.86$ & $\leq 7.62$ & - & $17^{\prime \prime} .5 \times 3^{\prime \prime} .0,-63^{\circ}$ \\
\hline $1.90-1.95$ & $\leq 7.89$ & $\leq 7.95$ & - & $16^{\prime \prime} 6 \times 3^{\prime \prime} .2,-63^{\circ}$ \\
\hline $1.95-2.00$ & $\leq 9.42$ & $\leq 8.48$ & - & $16^{\prime \prime} .8 \times 3^{\prime \prime} .1,-64^{\circ}$ \\
\hline
\end{tabular}

Notes. The time in the first column refers to the time in hours after the start of the observation (November 30, 2013, at 19:15:00). The polarisation fraction is shown for the times where both Stokes I and V are detected. When only Stokes I or V are detected, $3 \sigma$ upper or lower limits, respectively, are given for the polarisation fraction. The dimensions and position angle of the synthesised beam in the image at each time are shown in the final column. 
A. Feeney-Johansson et al.: Detection of coherent low-frequency radio bursts from weak-line T Tauri stars

Table D.3. Flux densities of LkCa 4 in Stokes I and V that were used in the light curve in Fig. 5.

\begin{tabular}{|c|c|c|c|c|}
\hline $\begin{array}{l}\text { Time } \\
\text { (hrs) }\end{array}$ & $\begin{array}{l}\text { Stokes I } \\
(\mathrm{mJy})\end{array}$ & $\begin{array}{c}\text { Stokes V } \\
(\mathrm{mJy})\end{array}$ & $\begin{array}{l}\text { Pol. Fraction } \\
(\%)\end{array}$ & Beam \\
\hline $0-0.5$ & $\leq 4.38$ & $\leq 3.21$ & - & $26^{\prime \prime} .8 \times 3^{\prime \prime} .5,-46^{\circ}$ \\
\hline $0.5-1.0$ & $\leq 3.24$ & $\leq 2.61$ & - & $25^{\prime \prime} .1 \times 3^{\prime \prime} .5,-50^{\circ}$ \\
\hline $1.0-1.5$ & $4.99 \pm 0.99$ & $3.30 \pm 0.70$ & $64_{-16}^{+18}$ & $23^{\prime \prime} .8 \times 3^{\prime \prime} \cdot 3,-55^{\circ}$ \\
\hline $1.5-2.0$ & $4.30 \pm 0.76$ & $3.76 \pm 0.61$ & $80_{-15}^{+13}$ & $22^{\prime \prime} \cdot 7 \times 3^{\prime \prime} .1,-59^{\circ}$ \\
\hline $2.0-2.5$ & $2.76 \pm 0.68$ & $3.00 \pm 0.56$ & $84_{-16}^{+11}$ & $21^{\prime \prime} \cdot 7 \times 2^{\prime \prime} \cdot 9,-65^{\circ}$ \\
\hline $2.5-3.0$ & $\leq 1.89$ & $\leq 1.47$ & - & $20^{\prime \prime} .8 \times 2^{\prime \prime} .9,-71^{\circ}$ \\
\hline $3.0-3.5$ & $\leq 2.01$ & $\leq 1.47$ & - & $19^{\prime \prime} .6 \times 3^{\prime \prime} .0,-77^{\circ}$ \\
\hline $3.5-4.0$ & $\leq 1.89$ & $\leq 1.44$ & - & $15^{\prime \prime} .2 \times 4^{\prime \prime} .9,66^{\circ}$ \\
\hline $4.0-4.5$ & $\leq 1.80$ & $\leq 1.50$ & - & $21^{\prime \prime} .2 \times 3^{\prime \prime} .8,84^{\circ}$ \\
\hline $4.5-5.0$ & $\leq 1.74$ & $\leq 1.44$ & - & $13^{\prime \prime} .4 \times 4^{\prime \prime} .0,67^{\circ}$ \\
\hline $5.0-5.5$ & $\leq 1.74$ & $\leq 1.47$ & - & $6^{\prime \prime} .2 \times 2^{\prime \prime} .3,-52^{\circ}$ \\
\hline $5.5-6.0$ & $2 . \overline{28} \pm 0.62$ & $\leq 1.53$ & $\leq 67$ & $9^{\prime \prime} .8 \times 3^{\prime \prime} .9,62^{\circ}$ \\
\hline $6.0-6.5$ & $\leq 1.83$ & $\leq 1.53$ & - & $7^{\prime \prime} .5 \times 33^{\prime \prime} 9,61^{\circ}$ \\
\hline $6.5-7.0$ & $3.95 \pm 0.60$ & $2.40 \pm 0.53$ & $60_{-15}^{+17}$ & $6^{\prime \prime} .5 \times 4^{\prime \prime} .0,60^{\circ}$ \\
\hline $7.0-7.5$ & $4.46 \pm 0.65$ & $3.90 \pm 0.56$ & $82_{-13}^{+11}$ & $6^{\prime \prime} 1 \times 4^{\prime \prime} .0,59^{\circ}$ \\
\hline $7.5-8.0$ & $\leq 2.25$ & $\leq 1.86$ & - & $6 ! .0 \times 44^{\prime \prime} 0,57^{\circ}$ \\
\hline
\end{tabular}

Notes. The time in the first column refers to the time in hours after the start of the observation (August 10, 2019, at 02:11:00). The polarisation fraction is shown for the times where both Stokes I and V are detected. When only Stokes I is detected, $3 \sigma$ upper limits are given for the polarisation fraction. The dimensions and position angle of the synthesised beam in the image at each time are shown in the final column. 